\title{
Replica approach to the KPZ equation with half Brownian motion initial condition
}

\author{
Takashi Imamura *, Tomohiro Sasamoto ${ }^{\dagger}$
}

November 15, 2018

\begin{abstract}
We consider the one-dimensional Kardar-Parisi-Zhang (KPZ) equation with half Brownian motion initial condition, studied previously through the weakly asymmetric simple exclusion process. We employ the replica Bethe ansatz and show that the generating function of the exponential moments of the height is expressed as a Fredholm determinant. From this the height distribution and its asymptotics are studied. Furthermore using the replica method we also discuss the multi-point height distribution. We find that some nice properties of the deformed Airy functions play an important role in the analysis.
\end{abstract}

\section{Introduction}

Surface growth phenomena appear widely in nature and have attracted much attention in non-equilibrium physics. In 1986, Kardar, Parisi and Zhang proposed a stochastic differential equation which describes surface growth with local interaction [1]. For onedimensional case, the KPZ equation is given by

$$
\frac{\partial h(x, t)}{\partial t}=\frac{\lambda}{2}\left(\frac{\partial h(x, t)}{\partial x}\right)^{2}+\nu \frac{\partial^{2} h(x, t)}{\partial x^{2}}+\sqrt{D} \eta(x, t) .
$$

Here $h(x, t)$ represents the height of the surface at position $x \in \mathbb{R}$ and time $t \geq 0$. The first term represents the effect of nonlinearity and the second one describes the smoothing effect of the surface. $\eta(x, t)$ represents randomness described by the Gaussian white noise with covariance,

$$
\left\langle\eta(x, t) \eta\left(x^{\prime}, t^{\prime}\right)\right\rangle=\delta\left(x-x^{\prime}\right) \delta\left(t-t^{\prime}\right) .
$$

${ }^{*}$ Research Center for Advanced Science and Technology, The University of Tokyo, E-mail: imamura@jamology.rcast.u-tokyo.ac.jp

†Department of mathematics and informatics, Chiba University, E-mail: sasamoto@math.s.chibau.ac.jp 
The parameters $\lambda, \nu, D$ determine their strengths. For the one-dimensional KPZ equation (1.1), it has been shown by the dynamical renormalization group that the height fluctuation scales as $O\left(t^{1 / 3}\right)$ as $t$ goes to infinity [1]. The exponent $1 / 3$ coincides with the ones found in Monte Carlo simulations of many stochastic models of surface growth. The $\mathrm{KPZ}$ equation is accepted as a prototypical equation describing the universality class called the KPZ universality class.

Our understanding of the KPZ universality class has deepened in the last decade. Not merely the exponent but the height distribution functions have been computed based on intriguing connections with the random matrix theory [2]. For the totally asymmetric simple exclusion process(TASEP), which is an exactly solvable model in the KPZ universality class, the current distribution function for the step initial condition has been obtained exactly and it was found that in the long time limit, it converges to the GUE (Gaussian Unitary Ensemble) Tracy-Widom distribution function [5], the largest eigenvalue distribution in random hermitian matrix [2].

An interesting finding from the studies of distribution functions is that they detect the difference of initial conditions, which the scaling exponent of the height cannot. For example, in the surface growth model called polynuclear growth(PNG) model, it has been recognized that the GUE Tracy-Widom distribution describes the height distribution in the droplet growth [3] while in the flat initial condition, it is described by the GOE (Gaussian Orthogonal Ensemble) Tracy-Widom distribution [4]. Furthermore, generalizations of these results to multi-point distribution function have also been studied and the universal processes, the Airy 2 [6] and Airy 17,8$]$ processes have been obtained. For recent progresses of this topic, see $[9,10,11]$.

Since 2010, the studies on the KPZ equation and KPZ universality class have entered a new stage [12]. First as an experimental progress, high-accuracy measurements for the exponents and the height distribution in the KPZ growth problem using turbulent liquid crystal have been performed and the GUE Tracy-Widom distribution function was observed as well as the critical exponents [13]. Second, we have begun to understand exactly height distribution function of the KPZ equation itself. In $[14,15,16,17]$ the height distribution at a single position was computed for the narrow-wedge initial condition,

$$
\frac{\lambda}{2 \nu} h(x, t=0)=-\frac{|x|}{\delta}, \delta \rightarrow 0
$$

from which the surface grows to a parabolic shape. In the long-time limit, the fluctuation around the macroscopic shape is described by the GUE Tracy-Widom distribution. The analysis of $[14,15,16,17]$ is based on recent progress on the current distribution of the (partially) ASEP $[18,19]$ and a fact that, in the weak asymmetry limit, the stochastic time evolution of the current of the ASEP can be mapped to that of the height described by the KPZ equation [20]. For more recent developments, see [21, 22, 23, 24, 25, 26].

More direct approach without relying on the results on the ASEP has also been developed recently [27, 28, 29]. The idea was first proposed in [30], in which the author used the interesting relation that the exponential moment of the height is represented as the dynamics of the one-dimensional $\delta$-function Bose gas with attractive interaction, which 
is solved by the Bethe ansatz [31, 32]. In the original work [30], only the ground state contribution was considered and the dynamical exponent 1/3 was obtained. In [28, 29], the authors succeeded in taking into account the whole contribution of the eigenstates and obtained the Fredholm determinant representation of the generating function of the exponential moment for the narrow wedge initial condition (1.3). More recently a multi-point generating function is also discussed [33, 34].

The replica method is quite attractive since we expect to be able to calculate various quantities for the one-dimensional KPZ equation directly and easily. In particular, we expect it would be a powerful tool to understand the universality in the KPZ equation. There are two recent progresses in this direction: In [35], the authors discussed a process characterizing the renormalization fixed point of the KPZ universality class and the Fredholm determinant expression of its transition probability has been obtained. In [36], the authors have obtained the exact height distribution for the flat initial condition and clarified its convergence to the GOE Tracy-Widom distribution in the long time limit.

In this paper, we pursue the potential of the replica method by applying it to the half Brownian-motion initial condition depicted in Fig. 1. This is one of the typical spatially extended initial conditions and is written in terms of the single valued function $h(x, t)$ as

$$
\frac{\lambda}{2 \nu} h(x, t=0)= \begin{cases}x / \delta, \delta \rightarrow 0, & x<0, \\ \alpha B(x), & x \geq 0 .\end{cases}
$$

Here $B(x)$ represents the one-dimensional standard Brownian motion with $B(0)=0$ and $\alpha=(2 \nu)^{-3 / 2} \lambda D^{1 / 2}$. For this initial condition, the macroscopic shape expected by solving (1.1) without the noise term is

$$
h(x, t) \sim \begin{cases}-x^{2} / 2 \lambda t, & x \leq 0, \\ 0, & 0<x .\end{cases}
$$

The main interest in this paper is the fluctuation around this macroscopic shape. We expect that a one-dimensional Brownian motion describes the fluctuation in the positive region $(x>0)$ since it is known to be a stationary measure of the KPZ equation. On the other hand, in the negative region $(x<0)$ where the parabolic growth is observed the situation is the same as the narrow wedge case. Thus the fluctuation property around the origin shows a crossover behavior between the two typical growths.

This initial condition has already been considered in [38]. There the analysis is based on the result for the ASEP with step Bernoulli initial condition [37] and naturally leads to an expression for the height distribution in the form of contour integral. We take the replica method and mainly treat the generating function of the exponential moment. Accordingly our formula is somewhat different from the one in [38] and is expressed in terms only of real quantities. Furthermore the replica approach allows us to discuss the multi-point distribution function with the help of the factorization assumption by [33, 34] and properties of the deformed Airy functions discussed in Appendix.

This paper is arranged as follows. In the next section, we state our main results. Briefly explaining the relation between the KPZ equation and the $\delta$-Bose gas in Sec. 3, we give 


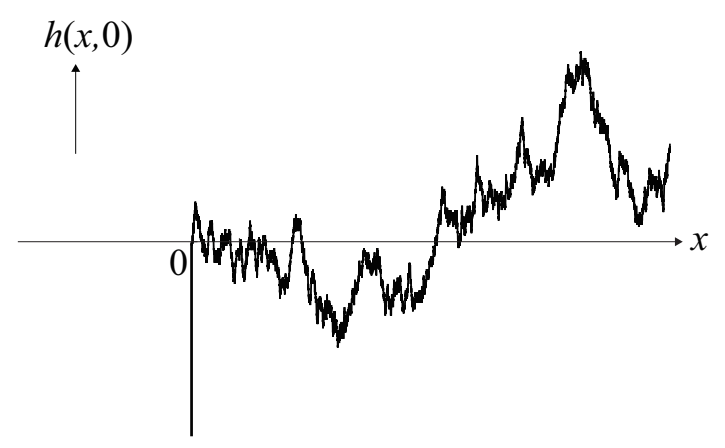

Figure 1: Half Brownian motion initial condition

a derivation of our main result, the Fredholm determinant expression of the generating function (Theorem 1 in Sec. 2) in Sec. 4. In Sec. 5, we discuss another expression of Theorem 1 stated as Proposition 2 in Sec. 2, which is useful for the compact expression of the height distribution function described by Theorem 3 in Sec. 2. In Sec. 6, we discuss the multi-point height distribution. In Sec. 7, we consider the interpretation of our result as free energy distribution of a directed polymer in random media. Concluding remarks are given in the last section.

\section{Model and main results}

\subsection{The generating function}

The KPZ equation (1.1) is in fact ill-defined as it is. As time goes on the height profile approaches the stationary one described by the Brownian motion, for which the non-linear term in the KPZ equation is not well-defined. A proper prescription is proposed in [20], which we follow here. In this scheme, one defines the height of the KPZ equation by

$$
h_{\nu, \lambda, D}(x, t)=\frac{2 \nu}{\lambda} \log \left(Z_{\nu, \lambda, D}(x, t)\right),
$$

using the solution $Z_{\nu, \lambda, D}(x, t)$ of the stochastic heat equation,

$$
\frac{\partial Z_{\nu, \lambda, D}(x, t)}{\partial t}=\nu \frac{\partial^{2} Z_{\nu, \lambda, D}(x, t)}{\partial x^{2}}+\frac{\lambda \sqrt{D}}{2 \nu} \eta(x, t) Z_{\nu, \lambda, D}(x, t),
$$

which is a well-defined stochastic partial differential equation of Itô-type. This is called the Cole-Hopf solution of the KPZ equation because the equation (2.2) is related to the $\mathrm{KPZ}$ equation through the (inverse of) the Cole-Hopf transformation (2.1). We will give more explanations in subsection 3.1 below. Hereafter we investigate the properties of these regularized quantities $h_{\nu, \lambda, D}(x, t)$ and $Z_{\nu, \lambda, D}(x, t)$ with the initial conditions (1.4). In the following we denote by $\langle\cdots\rangle$ the average over both $\eta(x, t)$ in the KPZ equation (1.1) and $B(x)$ in the initial condition (1.4). 
We are interested in the distribution of the height $h_{\nu, \lambda, D}(x, t)$. It is well established, known as the KPZ scaling, that the fluctuation of the height scales like $O\left(t^{1 / 3}\right)$ and nontrivial correlations are seen in the $x$ direction with scale $O\left(t^{2 / 3}\right)$ when $t$ is large. Let us define a parameter $\gamma_{t}$ which scales as $O\left(t^{1 / 3}\right)$ and a rescaled space coordinate $X$ by

$$
\gamma_{t}=\left(\alpha^{4} \nu t\right)^{\frac{1}{3}}, \quad x=\frac{2 \gamma_{t}^{2} X}{\alpha^{2}},
$$

with $\alpha$ given below (1.4). We introduce the scaled height $\tilde{h}_{t}(X)$ by

$$
\frac{\lambda}{2 \nu} h_{\nu, \lambda, D}\left(\frac{2 \gamma_{t}^{2} X}{\alpha^{2}}, t\right)=-\frac{\gamma_{t}^{3}}{12}-\gamma_{t} X^{2}+\gamma_{t} \tilde{h}_{t}(X)
$$

Here the second term corresponds to the macroscopic shape in (1.5); it is an interesting aspect of the KPZ equation that one has also to take into account the first term to focus on the height fluctuations.

To study the distribution, it is often useful to consider the generating function of the moments $\left\langle Z_{\nu, \lambda, D}(x, t)^{N}\right\rangle, N=0,1,2, \cdots$. We define $G_{\gamma_{t}}(s ; X)$ as

$$
G_{\gamma_{t}}(s ; X)=\sum_{N=0}^{\infty} \frac{\left(-e^{-\gamma_{t} s}\right)^{N}}{N !}\left\langle Z_{\nu, \lambda, D}^{N}\left(\frac{2 \gamma_{t}^{2} X}{\alpha^{2}}, t\right)\right\rangle e^{N \frac{\gamma_{t}^{3}}{12}+N \gamma_{t} X^{2}}=\left\langle e^{-e^{\gamma_{t}\left(\tilde{h}_{t}(X)-s\right)}}\right\rangle .
$$

By using the replica method, one can express the moment $\left\langle Z_{\nu, \lambda, D}(x, t)^{N}\right\rangle$ of our problem in the language of the $\delta$-function Bose gas, which is a well-known exactly solvable model. One can further perform the summation over $N$ to obtain the Fredholm determinant representation of the generating function.

Theorem $1 G_{\gamma_{t}}(s ; X)$ is expressed as the Fredholm determinant with the kernel acting on $L^{2}(\mathbb{R})$,

$$
G_{\gamma_{t}}(s ; X)=\operatorname{det}\left(1-P_{0} K_{X} P_{0}\right) .
$$

Here $P_{s}$ represents the projection onto $(s, \infty)$ and the kernel of $K_{X}$ is given by

$$
K_{X}\left(\omega_{j}, \omega_{k}\right)=\sum_{n=1}^{\infty}(-1)^{n-1} \int_{\mathbb{R}-i c_{n}} \frac{d q}{\pi} e^{-n\left(\omega_{j}+\omega_{k}\right)-2 i q\left(\omega_{j}-\omega_{k}\right)-\gamma_{t}^{3} n q^{2}+\frac{\gamma_{t}^{3}}{12} n^{3}-\gamma_{t} n s} \frac{\Gamma\left(i q-\frac{X}{\gamma_{t}}-\frac{n}{2}\right)}{\Gamma\left(i q-\frac{X}{\gamma_{t}}+\frac{n}{2}\right)},
$$

where $\Gamma(x)$ is the gamma function and $c_{n}$ satisfies $c_{n}>X / \gamma_{t}+n / 2$.

Here for an operator on $L^{2}(\mathbb{R})$ with kernel $K(x, y)$, the Fredholm determinant is defined by

$$
\operatorname{det}(1-K)=\sum_{M=0}^{\infty} \frac{(-1)^{M}}{M !} \int_{-\infty}^{\infty} d x_{1} \cdots \int_{-\infty}^{\infty} d x_{M} \operatorname{det}\left(K\left(x_{j}, x_{k}\right)\right)_{j, k=1}^{M}
$$


where the right hand side is assumed to converge.

A derivation of this result will be given in Sec. 4 after the explanation of the relation of $N$ th moment of $Z_{\nu, \lambda, D}(x, t)$ with $N$ particle dynamics of the $\delta$-function Bose gas in Sec. 3 . For the narrow wedge case (1.3) where $Z_{\nu, \lambda, D}(x, 0)=\delta(x)$, the corresponding generating function has been obtained in the same form as $(2.6)[28,29]$. But the kernel $K_{X}\left(\omega_{j}, \omega_{k}\right)$ is replaced by

$$
K_{\mathrm{nw}}\left(\omega_{j}, \omega_{k}\right)=\sum_{n=1}^{\infty}(-1)^{n-1} \int_{-\infty}^{\infty} \frac{d q}{\pi} e^{-n\left(\omega_{j}+\omega_{k}\right)-2 i q\left(\omega_{j}-\omega_{k}\right)-\gamma_{t}^{3} n q^{2}+\frac{\gamma_{t}^{3}}{12} n^{3}-\gamma_{t} n s},
$$

which is independent of the position $X$.

One can replace the kernel by the one written in the form of products of two deformed Airy functions.

Proposition 2 In (2.6), the kernel $P_{0} K_{X} P_{0}$ can be replaced by $P_{0} \bar{K}_{X} P_{0}$ where

$$
\bar{K}_{X}\left(\xi_{j}, \xi_{k}\right)=\int_{-\infty}^{\infty} d y \operatorname{Ai}^{\Gamma}\left(\xi_{j}+y, \frac{1}{\gamma_{t}},-\frac{X}{\gamma_{t}}\right) \operatorname{Ai}_{\Gamma}\left(\xi_{k}+y, \frac{1}{\gamma_{t}},-\frac{X}{\gamma_{t}}\right) \frac{e^{\gamma_{t} y}}{e^{\gamma_{t} y}+e^{\gamma_{t} s}}
$$

Here $\operatorname{Ai}^{\Gamma}(a, b, c), \operatorname{Ai}_{\Gamma}(a, b, c)$ are defined by

$$
\begin{aligned}
& \operatorname{Ai}^{\Gamma}(a, b, c)=\frac{1}{2 \pi} \int_{\Gamma_{i \frac{c}{b}}} d z e^{i z a+i \frac{z^{3}}{3}} \Gamma(i b z+c), \\
& \operatorname{Ai}_{\Gamma}(a, b, c)=\frac{1}{2 \pi} \int_{-\infty}^{\infty} d z e^{i z a+i \frac{z^{3}}{3}} \frac{1}{\Gamma(-i b z+c)} .
\end{aligned}
$$

In (2.11), $\Gamma_{z_{p}}$ represents the contour from $-\infty$ to $\infty$ and, along the way, passing below the pole $z_{p}=i c / b$.

The functions $\operatorname{Ai}^{\Gamma}(a, b, c), \operatorname{Ai}_{\Gamma}(a, b, c)$ have appeared in [38]. Note that these become the ordinary Airy function if the gamma function factors in the integrand are eliminated in (2.11) and (2.12). For the narrow wedge case, the corresponding kernel is the one where $\operatorname{Ai}^{\Gamma}\left(x, 1 / \gamma_{t},-X / \gamma_{t}\right)$ and $\operatorname{Ai}_{\Gamma}\left(x, 1 / \gamma_{t},-X / \gamma_{t}\right)$ are replaced by the Airy function. To get the kernel in proposition 2 from that in Theorem 1, one has to find some generalizations of formulas utilized in $[28,29]$. They and a derivation of (2.10) will be given in Sec. 5. Some properties of the deformed Airy functions are summarized in Appendix.

\subsection{The height distribution function}

The information of all the moments is enough to extract that of the probability distribution function. Applying the discussions in $[29,33]$, we can find a formula of the height distribution from the generating function $G_{\gamma_{t}}(s, X)$. Let $F_{\gamma_{t}}(s ; X)$ be

$$
F_{\gamma_{t}}(s ; X)=\operatorname{Prob}\left(\frac{\lambda}{2 \nu} h(x, t)+\frac{\gamma_{t}^{3}}{12}+\gamma_{t} X^{2} \leq \gamma_{t} s\right)=\operatorname{Prob}\left(\tilde{h}_{t} \leq s\right) .
$$


By using the Fredholm determinant $(2.6), F_{\gamma_{t}}(s ; X)$ can be expressed as follows [29, 33].

$$
F_{\gamma_{t}}(s ; X)=1-\int_{-\infty}^{\infty} d u e^{-e^{\gamma_{t}(s-u)}} g_{\gamma_{t}}(u ; X) .
$$

Here

$$
g_{\gamma_{t}}(u ; X)=\frac{1}{2 \pi i}\left(\operatorname{det}\left(1-P_{0} K_{X}^{+} P_{0}\right)-\operatorname{det}\left(1-P_{0} K_{X}^{-} P_{0}\right)\right)
$$

where $K_{X}^{ \pm}(x, y)$ is the kernel (2.7) or (2.10) in which the term $e^{-s}$ is replaced by $-e^{u} \pm i \epsilon$ with $\epsilon>0$ being infinitesimal.

Using (2.10), and the relation $1 /(x \pm i \epsilon)=\mathcal{P}(1 / x) \mp i \pi \delta(x)$, where $\mathcal{P}$ denotes the Cauchy principal value, we can easily find $K_{X}^{ \pm}$in $(2.15)$ is represented as

$$
\begin{aligned}
K_{X}^{ \pm}\left(\xi_{j}, \xi_{k}\right)= & \mathcal{P} \int_{-\infty}^{\infty} d y \operatorname{Ai}^{\Gamma}\left(\xi_{j}+y, \frac{1}{\gamma_{t}},-\frac{X}{\gamma_{t}}\right) \operatorname{Ai}_{\Gamma}\left(\xi_{k}+y, \frac{1}{\gamma_{t}},-\frac{X}{\gamma_{t}}\right)\left(\frac{1}{1-e^{\gamma_{t}(u-y)}}\right) \\
& \mp i \pi \operatorname{Ai}^{\Gamma}\left(\xi_{j}+u, \frac{1}{\gamma_{t}},-\frac{X}{\gamma_{t}}\right) \operatorname{Ai}_{\Gamma}\left(\xi_{k}+u, \frac{1}{\gamma_{t}},-\frac{X}{\gamma_{t}}\right) .
\end{aligned}
$$

Substituting this expression to (2.15) and using basic properties of determinant, we eventually arrive at the expression in terms of the Fredholm determinant.

\section{Theorem 3}

$$
F_{\gamma_{t}}(s ; X)=1-\int_{-\infty}^{\infty} d u e^{-e^{\gamma_{t}(s-u)}} g_{\gamma_{t}}(u ; X)
$$

Here $g_{\gamma_{t}}(u ; X)$ is expressed as a difference of two Fredholm determinants,

$$
g_{\gamma_{t}}(u ; X)=\operatorname{det}\left(1-P_{u}\left(B_{\gamma_{t}}^{\Gamma}-P_{\mathrm{Ai}}^{\Gamma}\right) P_{u}\right)-\operatorname{det}\left(1-P_{u} B_{\gamma_{t}}^{\Gamma} P_{u}\right),
$$

where

$$
\begin{aligned}
B_{\gamma_{t}}^{\Gamma}\left(\xi_{1}, \xi_{2}\right)= & \int_{0}^{\infty} d y \operatorname{Ai}^{\Gamma}\left(\xi_{1}+y, \frac{1}{\gamma_{t}},-\frac{X}{\gamma_{t}}\right) \operatorname{Ai}_{\Gamma}\left(\xi_{2}+y, \frac{1}{\gamma_{t}},-\frac{X}{\gamma_{t}}\right) \\
& +\int_{0}^{\infty} d y \frac{1}{e^{\gamma_{t} y}-1}\left(\operatorname{Ai}^{\Gamma}\left(\xi_{1}+y, \frac{1}{\gamma_{t}},-\frac{X}{\gamma_{t}}\right) \operatorname{Ai}_{\Gamma}\left(\xi_{2}+y, \frac{1}{\gamma_{t}},-\frac{X}{\gamma_{t}}\right)\right. \\
& \left.\quad-\operatorname{Ai}^{\Gamma}\left(\xi_{1}-y, \frac{1}{\gamma_{t}},-\frac{X}{\gamma_{t}}\right) \operatorname{Ai}_{\Gamma}\left(\xi_{2}-y, \frac{1}{\gamma_{t}},-\frac{X}{\gamma_{t}}\right)\right), \\
P_{\mathrm{Ai}}^{\Gamma}\left(\xi_{1}, \xi_{2}\right)= & \operatorname{Ai}^{\Gamma}\left(\xi_{1}, \frac{1}{\gamma_{t}},-\frac{X}{\gamma_{t}}\right) \operatorname{Ai}_{\Gamma}\left(\xi_{2}, \frac{1}{\gamma_{t}},-\frac{X}{\gamma_{t}}\right) .
\end{aligned}
$$

In [38], another expression using a contour integral was obtained whereas ours (2.17) are represented as the convolution with the Gumbel distribution. For numerical analysis, 


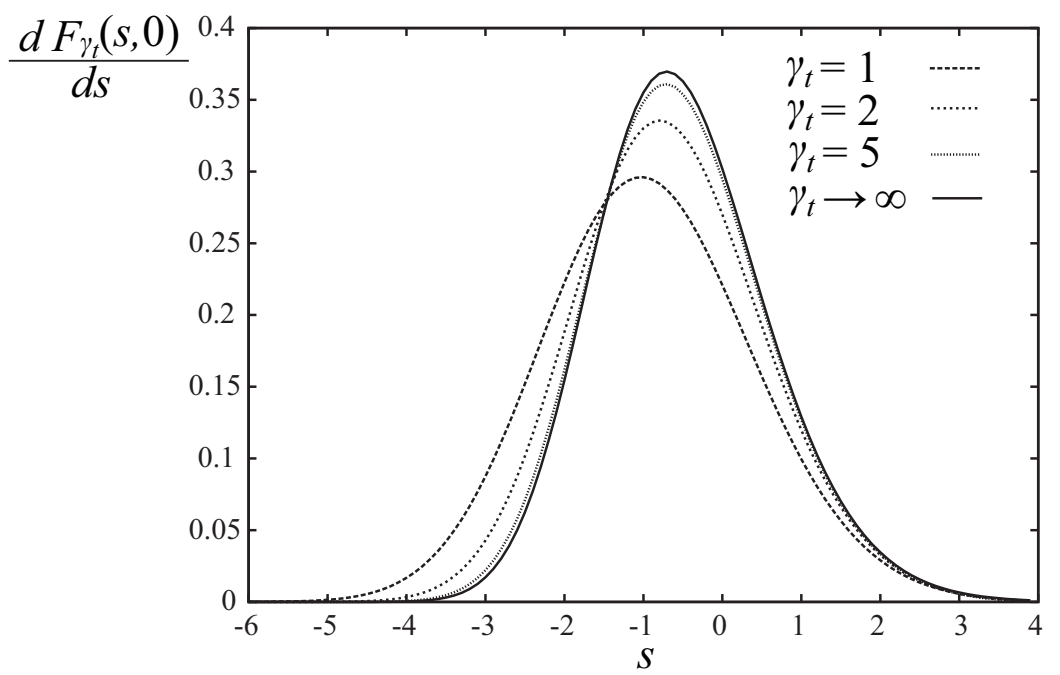

Figure 2: The probability density functions at the origin $X=0$. The three cases for finite $t^{\prime}$ 's $\left(\gamma_{t}=1,2,5\right)$ are depicted as the dashed lines while the solid line corresponds to the $\gamma_{t} \rightarrow \infty$ limit $($ see $(2.22))$.

this form would be convenient. Actually, from this expression, we can readily draw the picture of the probability density function $d F_{\gamma_{t}}(s ; X) / d s$ as in Fig. 2. In this figure, the graphs are drawn approximating the Fredholm determinant by a finite dimensional matrix determinant using a simple discretization. For more precise estimation, the method in [39, $40]$ is available and actually it was applied to the narrow wedge case [41].

The distribution function (2.17) has a similar form as the narrow wedge case obtained in $[14,15,16,17]$ : If we replace the functions $\operatorname{Ai}^{\Gamma}(x)$ and $\operatorname{Ai}_{\Gamma}(x)$ by the ordinary Airy function in (2.19) and (2.20), the distribution function (2.17) becomes the one for the narrow wedge initial condition.

We also consider the long-time limit $(t \rightarrow \infty)$ of the distribution function. Let us remember a basic fact that the probability distribution function is in general written as the expectation, $\operatorname{Prob}(X \leq s)=\mathbb{E}(\Theta(s-X))$, where $\Theta(y)$ is the step function, $\Theta(y):=$ $1(y \geq 0), 0(y<0)$. Noticing $\lim _{a \rightarrow \infty} \exp \left[-e^{-a x}\right]=\Theta(x)$, we take the $t \rightarrow \infty$ limit in $(2.5)$ and see

$$
\lim _{\gamma_{t} \rightarrow \infty} \operatorname{Prob}\left(\tilde{h}_{t} \leq s\right)=\lim _{\gamma_{t} \rightarrow \infty} G_{\gamma_{t}}(s ; X) .
$$

Combining this relation with Theorem 1 and Proposition 2, one obtains

$$
\lim _{\gamma_{t} \rightarrow \infty} \operatorname{Prob}\left(\tilde{h}_{t} \leq s\right)=\operatorname{det}\left(1-P_{s} \mathcal{K}_{X} P_{s}\right),
$$

the kernel is given by

$$
\begin{aligned}
\mathcal{K}_{X}\left(\xi_{j}, \xi_{k}\right):= & \lim _{\gamma_{t} \rightarrow \infty} \bar{K}_{X}\left(\xi_{j}-s, \xi_{k}-s\right) \\
= & \int_{0}^{\infty} d y \operatorname{Ai}\left(\xi_{j}+y\right) \operatorname{Ai}\left(\xi_{k}+y\right) \\
& \quad+\operatorname{Ai}\left(\xi_{k}\right)\left(e^{-\frac{X^{3}}{3}+X \xi_{j}}-\int_{0}^{\infty} d y e^{-X y} \operatorname{Ai}\left(\xi_{j}+y\right)\right) .
\end{aligned}
$$


This distribution function was obtained in the study on the PNG model with an external source $[42,43]$ and the TASEP with step Bernoulli initial condition [44]. At $X=0$, another expression using the solution to the Painlevé differential equation was given in [45]. In Fig. 2 , we illustrate the picture of the probability density function at the origin $X=0$ as a solid curve. The derivation of (2.22) will be given in Sec. 6 including the discussion of the multi-point height distribution.

\subsection{Multi-point distribution function}

The replica approach allows us to discuss multi-point height distribution function. We introduce the $n$-point generating function

$$
G_{\gamma_{t}}\left(\{s\}_{n},\{X\}_{n}\right)=\left\langle e^{-\sum_{j=1}^{n} e^{\gamma_{t}\left(\tilde{h}_{t}\left(X_{j}\right)-s_{j}\right)}}\right\rangle,
$$

where we abbreviated $s_{1}, \cdots, s_{n}$ and $X_{1}, \cdots, X_{n}$ as $\{s\}_{n}$ and $\{X\}_{n}$ respectively and we set $X_{1}<X_{2}<\cdots<X_{n}$.

In $[33,34]$, the authors proposed the "factorization assumption" for the $n$-point generating function for the narrow wedge initial condition. In this paper, we show that if we employ the same approximation to the case of half Brownian motion initial condition, we obtain the following result:

$$
G_{\gamma_{t}}^{\sharp}\left(\{s\}_{n},\{X\}_{n}\right)=\operatorname{det}(1-Q),
$$

and the kernel $Q(x, y)$ is given by

$$
Q\left(u_{1}, u_{n+1}\right)=\int_{-\infty}^{\infty} d u_{2} \cdots d u_{n}\left\langle u_{1}\left|e^{\left(X_{1}-X_{2}\right) H}\right| u_{2}\right\rangle \cdots\left\langle u_{n}\left|e^{\left(X_{n}-X_{1}\right) H} L_{1}\right| u_{n+1}\right\rangle \Phi\left(\{u-s\}_{n}\right),
$$

where $H$ is the Airy Hamiltonian $H=-\frac{\partial^{2}}{\partial u^{2}}+u$, and

$$
\begin{aligned}
\Phi\left(\{x\}_{n}\right) & =\frac{\sum_{j=1}^{n} e^{-\gamma_{t} x_{j}}}{1+\sum_{j=1}^{n} e^{-\gamma_{t} x_{j}},} \\
L_{j}(x, y) & =\int_{0}^{\infty} d w \operatorname{Ai}_{\Gamma}\left(w+x, \frac{1}{\gamma_{t}},-\frac{X_{j}}{\gamma_{t}}\right) \mathrm{Ai}^{\Gamma}\left(w+y, \frac{1}{\gamma_{t}},-\frac{X_{j}}{\gamma_{t}}\right),
\end{aligned}
$$

with $j \geq 1$. In (2.25), we put the symbol $\sharp$ in order to represent explicitly the fact that this is an expression after using the factorization approximation. The factor $\left\langle x\left|e^{\left(X_{j}-X_{k}\right) H}\right| y\right\rangle$ in $(2.26)$ can be expressed as

$$
\left\langle x\left|e^{\left(X_{j}-X_{k}\right) H}\right| y\right\rangle=\int_{-\infty}^{\infty} d z e^{-t z} \operatorname{Ai}_{\Gamma}\left(x+z, \frac{1}{\gamma_{t}},-\frac{X_{j}}{\gamma_{t}}\right) \operatorname{Ai}^{\Gamma}\left(y+z, \frac{1}{\gamma_{t}},-\frac{X_{k}}{\gamma_{t}}\right),
$$


see (A.1). For the one-point case $n=1$, however, the method in [33, 34] is exact. Actually, when $n=1$, we find the Fredholm determinant is equivalent to the kernel (2.10): In the one-point case, the kernel (2.26) becomes

$$
Q\left(u_{1}, u_{2}\right)=L_{1}\left(u_{1}, u_{2}\right) \Phi\left(u_{1}-s_{1}\right)
$$

We divide it into $Q=Q_{1} Q_{2}$, where

$$
\begin{aligned}
& Q_{1}(u, w)=\Phi(u-s) \mathrm{Ai}^{\Gamma}\left(w+u, \frac{1}{\gamma_{t}},-\frac{X_{1}}{\gamma_{t}}\right) \chi_{0}(w), \\
& Q_{2}(w, u)=\chi_{0}(w) \mathrm{Ai}_{\Gamma}\left(w+u, \frac{1}{\gamma_{t}},-\frac{X_{1}}{\gamma_{t}}\right)
\end{aligned}
$$

where $\chi_{s}(\xi)=1(s \leq \xi<\infty), 0(-\infty<\xi<s)$ and notice the property of the Fredholm determinant $\operatorname{det}\left(1-Q_{1} Q_{2}\right)=\operatorname{det}\left(1-Q_{2} Q_{1}\right)$. We easily find that $Q_{2} Q_{1}\left(\xi_{1}, \xi_{2}\right)=$ $\chi_{0}\left(\xi_{2}\right) \bar{K}_{X}\left(\xi_{2}, \xi_{1}\right) \chi_{0}\left(\xi_{2}\right)$, where $\bar{K}_{X}\left(\xi_{2}, \xi_{1}\right)$ is given in $(2.10)$.

Because an approximation is involved in the derivation, the expression (2.25) is most likely not exact when $n \geq 2$. A validity of employing this approximation is that, for the narrow wedge case, the generating function becomes the multi-point distribution function for the Airy $_{2}$ process in the long-time limit which is expected from the universality. In this paper we will see that the situation is similar for the case of the half Brownian motion initial condition. In the long-time limit, the multi-point generating function is equivalent to the multi-point height distribution function,

$$
\lim _{t \rightarrow \infty} G_{\gamma_{t}}\left(\{s\}_{n},\{X\}_{n}\right)=\lim _{t \rightarrow \infty} \operatorname{Prob}\left(\tilde{h}_{t}\left(X_{j}\right) \leq s_{j}, j=1, \cdots, n\right) .
$$

From (2.25), we find

$$
\begin{aligned}
& \lim _{t \rightarrow \infty} G_{\gamma_{t}}^{\sharp}\left(\{s\}_{n},\{X\}_{n}\right)=\operatorname{det}\left(1-P_{s} \mathcal{K}_{12} P_{s}\right) \\
& :=\sum_{M=0}^{\infty} \frac{(-1)^{M}}{M !} \prod_{i=1}^{M}\left(\sum_{n_{i}=1}^{n} \int_{-\infty}^{\infty} d \xi_{i} \chi_{s_{n_{i}}}\left(\xi_{i}\right)\right) \operatorname{det}\left(\mathcal{K}_{12}\left(X_{n_{j}}, \xi_{j} ; X_{n_{k}}, \xi_{k}\right)\right)_{j, k=1}^{M}
\end{aligned}
$$

where $\chi_{s}(\xi)$ is defined below $(2.32)$ and

$$
\begin{aligned}
& \mathcal{K}_{12}\left(X_{1}, \xi_{1} ; X_{2}, \xi_{2}\right)=\mathcal{K}_{2}\left(X_{1}, \xi_{1} ; X_{2}, \xi_{2}\right) \\
& \quad+\operatorname{Ai}\left(\xi_{2}\right)\left(e^{-\frac{X_{1}^{3}}{3}+X_{1} \xi_{1}}-\int_{0}^{\infty} d y e^{-X_{1} y} \operatorname{Ai}\left(\xi_{2}+y\right)\right), \\
& \mathcal{K}_{2}\left(X_{j}, \xi_{j} ; X_{k}, \xi_{k}\right)= \begin{cases}\int_{0}^{\infty} d y e^{-w\left(X_{j}-X_{k}\right)} \operatorname{Ai}\left(\xi_{j}+w\right) \operatorname{Ai}\left(\xi_{k}+w\right), & j \geq k, \\
-\int_{-\infty}^{0} d y e^{-w\left(X_{j}-X_{k}\right)} \operatorname{Ai}\left(\xi_{j}+w\right) \operatorname{Ai}\left(\xi_{k}+w\right), & j<k .\end{cases}
\end{aligned}
$$

The Fredholm determinant with this kernel has appeared in the PNG model and TASEP [42, $43,44]$. Note that for the one-point case $n=1$, it reduces to $(2.22)$. 
As pointed out in the Conjecture 9 in [38], it is plausible that in the long-time limit, the Fredholm determinant $\operatorname{det}\left(1-P_{s} \mathcal{K}_{12} P_{s}\right)$ describes the multi-point distribution considering the exact result of the one-point case. Thus (2.34) strongly suggests that the factorization approximation becomes exact in the long time limit in both narrow wedge and half Brownian motion initial conditions. In Sec. 6, we briefly discuss the derivation of these two equations (2.25) and (2.34).

\section{$3 \quad \mathrm{KPZ}$ equation and $\delta$-Bose gas}

\subsection{Feynman-Kac formula}

In subsection 2.1, we mentioned that the KPZ equation (1.1) is not well-defined as it is, and defined its solution by (2.1) and (2.2). Here we explain this using a limit of a modified version of the KPZ equation,

$$
\frac{\partial h_{\kappa}(x, t)}{\partial t}=\frac{\lambda}{2}\left(\frac{\partial h_{\kappa}(x, t)}{\partial x}\right)^{2}+\nu \frac{\partial^{2} h_{\kappa}(x, t)}{\partial x^{2}}+\sqrt{D} \eta_{\kappa}(x, t)-\frac{1}{2}\left(\frac{\lambda \sqrt{D}}{2 \nu}\right)^{2} C_{\kappa}(0) .
$$

This looks almost the same as the original (1.1), but there are two differences. First $\eta_{\kappa}(x, t)$ is still a Gaussian noise but its covariance in $x$-direction is now smeared as

$$
\left\langle\eta_{\kappa}(x, t) \eta_{\kappa}\left(x^{\prime}, t^{\prime}\right)\right\rangle=C_{\kappa}\left(x-x^{\prime}\right) \delta\left(t-t^{\prime}\right),
$$

where $C_{\kappa}(x)$ is written as $C_{\kappa}(x)=\kappa C(\kappa x)$ and $C(x)$ is a smooth, even and positive function

such that $\int_{-\infty}^{\infty} C(x)=1$. Second, the constant velocity term $-(\lambda \sqrt{D} / 2 \nu)^{2} C_{\kappa}(0) / 2$ is added. Note that the above properties of $C_{\kappa}$ imply $\lim _{\kappa \rightarrow \infty} C_{\kappa}(x)=\delta(x)$ so that the noise $\eta_{\kappa}$ tends to the white noise in the original KPZ equation. An apparent problem is that the additional term in (3.1) diverges as $\delta(0)$ but in fact this term is necessary for considering a meaningful limit of the equation (3.1). To see this let us apply the Cole-Hopf transformation,

$$
Z_{\kappa}(x, t)=\exp \left(\frac{\lambda}{2 \nu} h_{\kappa}(x, t)\right)
$$

to (3.1). By Itô's formula (3.1) becomes

$$
\frac{\partial Z_{\kappa}(x, t)}{\partial t}=\nu \frac{\partial^{2} Z_{\kappa}(x, t)}{\partial x^{2}}+\frac{\lambda \sqrt{D}}{2 \nu} \eta_{\kappa}(x, t) Z_{\kappa}(x, t),
$$

which is a well-defined stochastic differential equation of Itô-type. Clearly the solution $Z_{\kappa}(x, t)$ to this equation converges to that $Z_{\nu, \lambda, D}(x, t)$ of $(2.2)$ as $\kappa \rightarrow \infty$ [20]. Hence the Cole-Hopf solution (2.1) with (2.2) can be interpreted as a limit of the solution to the equation (3.1):

$$
Z_{\nu, \lambda, D}(x, t)=\lim _{\kappa \rightarrow \infty} Z_{\kappa}(x, t) .
$$


Note that if the constant velocity term is absent in (3.1), there appears an additional term in (3.4), or the equation (3.4) should be understood as representing a stochastic differential equation of Stratonovich type, which is not well-defined in the limit $\kappa \rightarrow \infty$.

The solution of (3.4) can be represented as the Feynman-Kac formula [46],

$$
Z_{\kappa}(x, t)=\mathbb{E}_{x}\left(\exp \left[\frac{\lambda \sqrt{D}}{2 \nu} \int_{0}^{t} \eta_{\kappa}(b(2 \nu s), t-s) d s\right] Z_{\kappa}(b(t), 0)\right) e^{-\frac{1}{2}\left(\frac{\lambda \sqrt{D}}{2 \nu}\right)^{2} C_{\kappa}(0) t}
$$

where $\mathbb{E}_{x}$ represents the averaging over the standard Brownian motion $b(s), 0<s<t$ with $b(0)=x$ and the initial condition is

$$
Z_{\kappa}(x, t=0)= \begin{cases}0, & x<0 \\ e^{\alpha B(x)}, & x \geq 0\end{cases}
$$

Here $B(x)$ represents the one-dimensional Brownian motion with $B(0)=0$. Some readers may find the following path-integral expression more intuitive.

$$
Z_{\kappa}(x, t)=\int_{0}^{\infty} d y \int_{x(0)=y}^{x(t)=x} D[x(\tau)] \exp \left(-S[x(\tau)]+\alpha B(y)-\frac{1}{2}\left(\frac{\lambda \sqrt{D}}{2 \nu}\right)^{2} C_{\kappa}(0) t\right),
$$

where the action $S[x(\tau)]$ is given by

$$
S[x(\tau)]=\int_{0}^{t} d \tau\left(\frac{1}{4 \nu}\left(\frac{d x(\tau)}{d \tau}\right)^{2}-\frac{\lambda \sqrt{D}}{2 \nu} \eta_{\kappa}(x(\tau), \tau)\right) .
$$

We find that $Z_{\nu, \lambda, D}(x, t)$ defined in $(2.1)$ is written in terms of $Z_{\frac{1}{2}, 1,1}(x, t)$ with specific parameters $\nu=1 / 2, \lambda=D=1$ as follows. First using the Feynman-Kac formula (3.6), one has

$$
\begin{aligned}
& Z_{\nu, \lambda, D}\left(x, \frac{t}{2 \nu}\right) \\
& =\lim _{\kappa \rightarrow \infty} \mathbb{E}_{x}\left(\exp \left[\frac{\lambda \sqrt{D}}{2 \nu} \int_{0}^{\frac{t}{2 \nu}} \eta_{\kappa}\left(b(2 \nu s), \frac{t}{2 \nu}-s\right) d s-\frac{1}{2}\left(\frac{\lambda \sqrt{D}}{2 \nu}\right)^{2} C_{\kappa}(0) \frac{t}{2 \nu}\right] Z^{(0)}(b(t), \alpha)\right),
\end{aligned}
$$

where we rewrite $Z_{\kappa}(x, 0)$ as $Z^{(0)}(x, \alpha)$ in order to represent explicitly the dependence on $\alpha$ and the independence of $\kappa$. From the properties of $C_{\kappa}$ written below (3.2) we find the scaling relations of $C_{\kappa}$ and $\eta_{\kappa}(x, t)$,

$$
\begin{aligned}
& C_{\kappa}(x)=a C_{\kappa / a}(a x), \\
& \eta_{\kappa}(x, t)=(a b)^{1 / 2} \eta_{\kappa / a}(a x, b t),
\end{aligned}
$$


where $a, b \in \mathbb{R}$ and the equality (3.12) holds in the sense of distribution. By use of (3.12) with $a=1, b=1 / 2 \nu,(3.10)$ becomes

$$
\lim _{\kappa \rightarrow \infty} \mathbb{E}_{x}\left(\exp \left[\alpha \int_{0}^{t} \eta_{\kappa}(b(s), t-s) d s-\frac{\alpha^{2}}{2} C_{\kappa}(0) t\right] Z^{(0)}(b(t), \alpha)\right) .
$$

Next one remembers the scaling property of the Brownian motion,

$$
a b_{x}(s)=b_{a x}\left(a^{2} s\right)
$$

where the equality holds again in the sense of distribution and we put the initial position $x$ of $b(s)$ explicitly. By (3.12) and (3.14) with $a=\alpha^{2}, b=\alpha^{4}$, Eq. (3.13) can be rewritten as

$$
\lim _{\kappa \rightarrow \infty} \mathbb{E}_{\alpha^{2} x}\left(\exp \left[\int_{0}^{\alpha^{4} t} \eta_{\kappa / \alpha^{2}}\left(b(s), \alpha^{4} t-s\right) d s-\frac{\alpha^{4}}{2} C_{\kappa / \alpha^{2}}(0) t\right] Z^{(0)}\left(\alpha^{-2} b\left(\alpha^{4} t\right), \alpha\right)\right) .
$$

At last noticing from (3.7) and (3.14) that

$$
Z^{(0)}\left(\alpha^{-2} x, \alpha\right)=Z^{(0)}(x, \alpha=1)
$$

we find (3.15) is nothing but $Z_{\frac{1}{2}, 1,1}\left(\alpha^{2} x, \alpha^{4} t\right)$.

Thus we have established

$$
\frac{\lambda}{2 \nu} h_{\nu, \lambda, D}\left(x, \frac{t}{2 \nu}\right)=h_{\frac{1}{2}, 1,1}\left(\alpha^{2} x, \alpha^{4} t\right) .
$$

In what follows we restrict our discussions to $h_{\frac{1}{2}, 1,1}(x, t)$ and $Z_{\frac{1}{2}, 1,1}(x, t)$ (hereafter we omit the indices $\left.\frac{1}{2}, 1,1\right)$. We remark that, for this special parameter values, (2.3) reads

$$
\gamma_{t}=\left(\frac{t}{2}\right)^{\frac{1}{3}}, \quad x=2 \gamma_{t}^{2} X
$$

\subsection{The $\delta$-function Bose gas}

Next we consider the replica partition function $\left\langle Z^{N}(x, t)\right\rangle(N=0,1,2, \cdots)$. First we perform the Gaussian average over $\eta_{\kappa}(x, t)$ using the path integral representation (3.8) and (3.2), to find

$$
\left\langle e^{N \int_{0}^{t} d \tau \eta(x, \tau)}\right\rangle=e^{\frac{1}{2} \sum_{j, k=1}^{N} C_{\kappa}\left(x_{j}-x_{k}\right)}
$$

The right hand side of this equation includes the self-interaction term $\sum_{j=k=1}^{N} C_{\kappa}\left(x_{j}-x_{k}\right)=$ $N C_{\kappa}(0)$, which becomes divergent in the limit $\kappa \rightarrow \infty$. This term, however, cancels out 
the last term in (3.8), and thus we can take the limit $\kappa \rightarrow \infty$. We obtain

$$
\begin{aligned}
& \left\langle Z^{N}(x, t)\right\rangle=\lim _{\kappa \rightarrow \infty}\left\langle Z_{\kappa}^{N}(x, t)\right\rangle \\
& =\prod_{j=1}^{N} \int_{0}^{\infty} d y_{j} \int_{x_{j}(0)=y_{j}}^{x_{j}(t)=x} D\left[x_{j}(\tau)\right] \exp \left[-\int_{0}^{t} d \tau\left(\sum_{j=1}^{N} \frac{1}{2}\left(\frac{d x_{j}(\tau)}{d \tau}\right)^{2}-\sum_{j \neq k=1}^{N} \delta\left(x_{j}(\tau)-x_{k}(\tau)\right)\right)\right] \\
& \quad \times\left\langle\exp \left(\sum_{k=1}^{N} B\left(y_{k}\right)\right)\right\rangle
\end{aligned}
$$

where $\langle\cdots\rangle$ in the last factor indicates the remaining average over the Brownian motion $B(y)$.

The right hand side of this equation represents the imaginary-time dynamics of the $\delta$-function Bose gas with attractive interaction with the Hamiltonian $H_{N}$,

$$
H_{N}=-\frac{1}{2} \sum_{j=1}^{N} \frac{\partial^{2}}{\partial x_{j}^{2}}-\frac{1}{2} \sum_{j \neq k}^{N} \delta\left(x_{j}-x_{k}\right),
$$

in terms of which the replica partition function $\left\langle Z^{N}(x, t)\right\rangle$ can be written as

$$
\left\langle Z^{N}(x, t)\right\rangle=\left\langle x\left|e^{-H_{N} t}\right| \Phi\right\rangle .
$$

Here $\langle x|$ represents the state with all $N$ particles being at the position $x$ and the $|\Phi\rangle$ the initial state of the $\delta$-function Bose gas. One can perform the average over the Brownian motion $B(y)$ and the dependence of $|\Phi\rangle$ on $x_{1}, \ldots, x_{N}$ can be explicitly calculated as

$$
\begin{aligned}
& \left\langle x_{1}, \cdots, x_{N} \mid \Phi\right\rangle \\
= & \frac{1}{N !} \sum_{P \in S_{N}}\left\langle\exp \left(\sum_{k=1}^{N} B\left(x_{P(k)}\right)\right)\right\rangle \\
= & \sum_{P \in S_{N}} \prod_{j=1}^{N}\left(\int_{-\infty}^{\infty} d z_{j} \frac{e^{-z_{j}-\frac{\left(z_{j}-z_{j-1}\right)^{2}}{2\left(x_{P(j)}-x_{P(j-1)}\right)}}}{\sqrt{2 \pi\left(x_{P(j)}-x_{P(j-1)}\right)}} \Theta\left(x_{P(j)}-x_{P(j-1)}\right)\right) \\
= & \sum_{P \in S_{N}} \prod_{j=1}^{N} e^{\frac{1}{2}(2 N-2 j+1) x_{P(j)} \Theta\left(x_{P(j)}-x_{P(j-1)}\right) .}
\end{aligned}
$$

Here $S_{N}$ denotes the set of permutations of order $N, \Theta(x)$ is the step function and we set $x_{P(0)}=0$. Since we are considering a Boson system, the above function is taken to be symmetric in $x_{1}, \cdots, x_{N}$.

The eigenvalues and eigenfunctions of the $\delta$-Bose gas can be constructed by using the Bethe ansatz $[27,28,29,31,32]$. Let $\left|\Psi_{z}\right\rangle$ and $E_{z}$ be the eigenstate and its eigenvalue of $H_{N}$,

$$
H_{N}\left|\Psi_{z}\right\rangle=E_{z}\left|\Psi_{z}\right\rangle
$$


By the Bethe ansatz, they are given as

$$
\left\langle x_{1}, \cdots, x_{N} \mid \Psi_{z}\right\rangle=C_{z} \sum_{P \in S_{N}} \operatorname{sgn} P \prod_{1 \leq j<k \leq N}\left(z_{P(j)}-z_{P(k)}+i \operatorname{sgn}\left(x_{j}-x_{k}\right)\right) \exp \left(i \sum_{l=1}^{N} z_{P(l)} x_{l}\right)
$$

where $C_{z}$ is the normalization constant, for which a formula is given in (3.27) below.

For the $\delta$-Bose gas with attractive interaction, the quasimomenta $z_{j}(1 \leq j \leq N)$ which label the state, are in general complex numbers. $z_{j}(1 \leq j \leq N)$ are divided into $M$ groups where $1 \leq M \leq N$. The $\alpha$ th group consists of $n_{\alpha}$ quasimomenta $z_{j}^{\prime} s$ which share the common real part $q_{\alpha}$. Note that $\sum_{\alpha=1}^{M} n_{\alpha}=N$. The quasimomenta in each group line up with regular intervals with unit length along the imaginary direction. Using $q_{\alpha}$ and $n_{\alpha}(1 \leq \alpha \leq M)$, we represent $z_{j}(1 \leq j \leq N)$ as

$$
z_{j}=q_{\alpha}-\frac{i}{2}\left(n_{\alpha}+1-2 r_{\alpha}\right), \text { for } j=\sum_{\beta=1}^{\alpha-1} n_{\beta}+r_{\alpha},
$$

where $1 \leq \alpha \leq M$ and $1 \leq r_{\alpha} \leq n_{\alpha}$. The normalization constant $C_{z}$, which is taken to be a positive real number, and the eigenvalue $E_{z}$ are given by [28]

$$
\begin{aligned}
C_{z} & =\left(\frac{\prod_{\alpha=1}^{M} n_{\alpha}}{N !} \prod_{1 \leq j<k \leq N} \frac{1}{\left|z_{j}-z_{k}-i\right|^{2}}\right)^{1 / 2}, \\
E_{z} & =\frac{1}{2} \sum_{j=1}^{N} z_{j}^{2}=\frac{1}{2} \sum_{\alpha=1}^{M} n_{\alpha} q_{\alpha}^{2}-\frac{1}{24} \sum_{\alpha=1}^{M}\left(n_{\alpha}^{3}-n_{\alpha}\right) .
\end{aligned}
$$

There is a problem of completeness of these states. This has not been resolved rigorously yet but the fact that one can recover the height distribution of the KPZ equation for the narrow wedge initial condition is a strong affirmative evidence. Here we proceed assuming its validity and will see the consistency with the computations based on the ASEP. This provides a further evidence that the above Bethe states are in fact complete.

\section{Generating function}

In this section, we give a derivation of the formula (2.6) in Theorem 1 . The replica partition function $\left\langle Z^{N}(x, t)\right\rangle(3.22)$ can be written as

$$
\left\langle Z^{N}(x, t)\right\rangle=\int_{-\infty}^{\infty} d y_{1} \cdots \int_{-\infty}^{\infty} d y_{N}\left\langle x\left|e^{-H_{N} t}\right| y_{1}, \cdots, y_{N}\right\rangle\left\langle y_{1}, \cdots, y_{N} \mid \Phi\right\rangle .
$$


Expanding the propagator $\left\langle x\left|e^{-H_{N} t}\right| y_{1}, \cdots, y_{N}\right\rangle$ by the Bethe eigenstates of the $\delta$-Bose gas (3.25), we have

$$
\begin{aligned}
\left\langle Z^{N}(x, t)\right\rangle= & \sum_{M=1}^{N} \frac{N !}{M !} \prod_{j=1}^{N} \int_{-\infty}^{\infty} d y_{j}\left(\int_{-\infty}^{\infty} \prod_{\alpha=1}^{M} \frac{d q_{\alpha}}{2 \pi} \sum_{n_{\alpha}=1}^{\infty}\right) \delta_{\sum_{\beta=1}^{M} n_{\beta}, N} \\
& \times e^{-E_{z} t}\left\langle x \mid \Psi_{z}\right\rangle\left\langle\Psi_{z} \mid y_{1}, \cdots, y_{N}\right\rangle\left\langle y_{1}, \cdots, y_{N} \mid \Phi\right\rangle .
\end{aligned}
$$

Here we want to perform the integrations over $y_{j},(1 \leq j \leq N)$,

$$
\prod_{j=1}^{N} \int_{-\infty}^{\infty} d y_{j}\left\langle\Psi_{z} \mid y_{1}, \cdots, y_{N}\right\rangle\left\langle y_{1}, \cdots, y_{N} \mid \Phi\right\rangle
$$

using (3.23) and (3.25). But this is not allowed for the moment because the integrations over $q_{\alpha},(1 \leq \alpha \leq M)$ must be performed before those over $y_{j},(1 \leq j \leq N)$. To see this explicitly, one uses (3.23) and notices the symmetry of the eigenfunction, $\left\langle\Psi_{z} \mid y_{P(1)}, \cdots, y_{P(N)}\right\rangle=\left\langle\Psi_{z} \mid y_{1}, \cdots, y_{N}\right\rangle$, to find that the right hand side of (4.2) is represented as

$$
\begin{aligned}
\left\langle Z^{N}(x, t)\right\rangle= & \sum_{M=1}^{N} \frac{N !}{M !} \prod_{j=1}^{N} \int_{y_{j-1}}^{\infty} d y_{j} \prod_{\alpha=1}^{M}\left(\int_{-\infty}^{\infty} \frac{d q_{\alpha}}{2 \pi} \sum_{n_{\alpha}=1}^{\infty}\right) \delta_{\sum_{\beta=1}^{M} n_{\beta}, N} \\
& \times e^{-E_{z} t}\left\langle x \mid \Psi_{z}\right\rangle\left\langle\Psi_{z} \mid y_{1}, \cdots, y_{N}\right\rangle \prod_{j=1}^{N} e^{\frac{1}{2}(2 N-2 j+1) y_{j}} .
\end{aligned}
$$

It is clear that the integrand on the right hand side of this equation is not integrable on $y_{j}(1 \leq j \leq M)$ due to the factor $\exp \left((2 N-2 j+1) y_{j}\right)$ while it is integrable on $q_{\alpha}(1 \leq \alpha \leq M)$ thanks to the factor $e^{-n_{\alpha} q_{\alpha}^{2} t / 2}$ in $e^{-E_{z} t}$.

\subsection{Deformation of contours}

To exchange the order of integrations, we notice

Lemma 4 In (4.4), we can deform the contour of $q_{\alpha}(1 \leq \alpha \leq M)$ to $\mathbb{R}-i c$ where $c$ is an arbitrary real constant.

Proof In (4.4), we change the variables from $q_{\alpha}$ to $u_{\alpha}(1 \leq j \leq M)$ such that

$$
u_{\alpha}=q_{\alpha}-q_{\alpha+1}, \quad(1 \leq \alpha \leq M-1), \quad u_{M}=q_{M} .
$$

The singularity in $u_{\alpha}$ of the integrand in (4.4) comes only from the factor $\left|C_{z}\right|^{2}$. Note that it depends only on $u_{1}, \cdots, u_{M-1}$ and is independent of $u_{M}$. Thus there are no poles of $u_{M}$ in (4.4), and we can deform the contour of $u_{M}$ to $\mathbb{R}-i c$. Noting that

$$
\prod_{\alpha=1}^{M-1} \int_{-\infty}^{\infty} \frac{d u_{\alpha}}{2 \pi} \times \int_{\mathbb{R}-i c} \frac{d u_{M}}{2 \pi}=\prod_{\alpha=1}^{M} \int_{\mathbb{R}-i c} \frac{d q_{\alpha}}{2 \pi},
$$

we find the statement holds. 
Using Lemma 4, we deform the contours of $q_{\alpha},(1 \leq \alpha \leq M)$ to $\mathbb{R}-i c$ where $c$ is a positive constant so large that the factor $\exp \left(-i \sum_{j=1}^{N} z_{P(j)}^{*} y_{j}+\frac{1}{2} \sum_{j=1}^{N}(2 N-2 j+1) y_{j}\right)$ in the integrand of (4.4) converge when $y_{j}>0$ goes to infinity. Here $z_{j}^{*}=q_{\alpha(j)}+$ $\frac{i}{2}\left(n_{\alpha(j)}+1-2 r(j)\right)$ but one has to be careful about the notation. This is a usual complex conjugate of $z_{j}$ before lemma 4, i.e., when $q_{\alpha(j)}$ is real. But now it is not because after the deformation of the contour, $q_{\alpha(j)}$ has an imaginary part.

After this deformation, we can perform the integrations of $y_{j}(1 \leq j \leq N)$ before those of $q_{\alpha}(1 \leq \alpha \leq M)$. Eq. (4.2) can now be expressed as

$$
\left\langle Z^{N}(x, t)\right\rangle=\sum_{M=1}^{N} \frac{1}{M !} \prod_{\alpha=1}^{M}\left(\int_{\mathbb{R}-i c} \frac{d q_{\alpha}}{2 \pi} \sum_{n_{\alpha}=1}^{\infty}\right) \delta_{\sum_{\beta=1}^{M} n_{\beta}, N}\left\langle x \mid \Psi_{z}\right\rangle\left\langle\Psi_{z} \mid \Phi\right\rangle e^{-E_{z} t} .
$$

Here $\left\langle x \mid \Psi_{z}\right\rangle$ is given by (3.25) with $x_{1}=\cdots=x_{N}=x$ and $\left\langle\Psi_{z} \mid \Phi\right\rangle$ is computed as

$$
\begin{aligned}
& \left\langle\Psi_{z} \mid \Phi\right\rangle=\prod_{j=1}^{N} \int_{-\infty}^{\infty} d y_{j}\left\langle\Psi_{z} \mid y_{1}, \cdots, y_{N}\right\rangle\left\langle y_{1}, \cdots, y_{N} \mid \Phi\right\rangle \\
& \left.=C_{z} \sum_{P \in S_{N}} \operatorname{sgn} P \prod_{l=1}^{N} \int_{y_{l-1}}^{\infty} d y_{l} e^{-i\left(z_{P(l)}^{*}+\frac{i}{2}(2 N-2 l+1)\right) y_{l}} \prod_{1 \leq j<k \leq N}\left(z_{P(j)}^{*}-z_{P(k)}^{*}+i\right)\right) \\
& =N ! C_{z} \sum_{P \in S_{N}} \operatorname{sgn} P \prod_{1 \leq j<k \leq N}\left(z_{P(j)}^{*}-z_{P(k)}^{*}+i\right) \prod_{l=1}^{N} \frac{-1}{-i\left(z_{P(N)}^{*}+\cdots+z_{P(N-l+1)}^{*}\right)+l^{2} / 2} .
\end{aligned}
$$

In (4.7), we take the imaginary part $c$ of $q_{\alpha}$ in such a way that we can perform the integrations of $y_{j}(1 \leq j \leq N)$ in $(4.8)$, i.e. $\operatorname{Re}\left(-i\left(z_{P(N)}^{*}+\cdots+z_{P(N-l+1)}^{*}\right)+l^{2} / 2\right)<0$ for any $l(1 \leq l \leq N)$. For example, if we fix $c$ such that $c>N / 2+\max _{\alpha} n_{\alpha} / 2$, the above condition is satisfied.

\subsection{Combinatorial identities}

For further analysis of the integrand in (4.7), we need two combinatorial identities for $\left\langle x \mid \Psi_{z}\right\rangle$ and $\left\langle\Psi_{z} \mid \Phi\right\rangle$. The first one is for $\left\langle x \mid \Psi_{z}\right\rangle$. One has

$$
\sum_{P \in S_{N}} \operatorname{sgn} P \prod_{1 \leq j<k \leq N}\left(w_{P(j)}-w_{P(k)}+i f(j, k)\right)=N ! \prod_{1 \leq j<k \leq N}\left(w_{j}-w_{k}\right)
$$

for any complex variables $w_{j}(1 \leq j \leq N)$ and $f(j, k)$. This identity was derived as Lemma 1 in [33].

The next one for the term $\left\langle\Psi_{z} \mid \Phi\right\rangle$ is 
Lemma 5 For any complex numbers $w_{j}(1 \leq j \leq N)$ and $a$,

$$
\begin{aligned}
& \sum_{P \in S_{N}} \operatorname{sgn} P \prod_{1 \leq j<k \leq N}\left(w_{P(j)}-w_{P(k)}+a\right) \prod_{m=1}^{N} \frac{1}{w_{P(N)}+\cdots+w_{P(N-m+1)}+m^{2} a / 2} \\
& =\prod_{1 \leq j<k \leq N}\left(w_{j}-w_{k}\right) \prod_{m=1}^{N} \frac{1}{w_{m}+a / 2} .
\end{aligned}
$$

Proof A similar identity appears in the context of ASEP with the step Bernoulli initial condition and has been proved in section III of [19]. Here we follow the same strategy using mathematical induction. Let us call the left hand side and the right hand side of (4.10) as $\psi_{N}$ and $\phi_{N}$ respectively, i.e.,

$$
\begin{aligned}
\psi_{N}\left(w_{1}, \cdots, w_{N}\right):= & \sum_{P \in S_{N}} \operatorname{sgn} P \\
& \prod_{1 \leq j<k \leq N}\left(w_{P(j)}-w_{P(k)}+a\right) \\
& \times \prod_{m=1}^{N} \frac{1}{w_{P(N)}+\cdots+w_{P(N-m+1)}+m^{2} a / 2}, \\
\phi_{N}\left(w_{1}, \cdots, w_{N}\right):= & \prod_{1 \leq j<k \leq N}\left(w_{j}-w_{k}\right) \prod_{m=1}^{N} \frac{1}{w_{m}+a / 2} .
\end{aligned}
$$

We want to show $\psi_{N}=\phi_{N}$. We easily see that it holds for the case $N=1$. Let us assume that it holds for $N-1$ case. In (4.11), we first sum over all permutations with $P(1)=l$ fixed and then sum over $l$. Noticing $\prod_{1<k \leq N}\left(w_{l}-w_{P(k)}+a\right)=\prod_{k(\neq l)}\left(w_{l}-w_{k}+a\right)$, we see

$$
\begin{aligned}
& \psi_{N}\left(w_{1}, \cdots, w_{N}\right) \\
& =\frac{1}{\sum_{j=1}^{N} w_{j}+N^{2} a / 2} \sum_{l=1}^{N}(-1)^{l+1} \prod_{j(\neq l)}\left(w_{l}-w_{j}+a\right) \psi_{N-1}\left(w_{1}, \cdots, w_{l-1}, w_{l+1}, \cdots, w_{N}\right) \\
& =\frac{1}{\sum_{j=1}^{N} w_{j}+N^{2} a / 2} \sum_{l=1}^{N}(-1)^{l+1} \prod_{j(\neq l)}\left(w_{l}-w_{j}+a\right) \phi_{N-1}\left(w_{1}, \cdots, w_{l-1}, w_{l+1}, \cdots, w_{N}\right),
\end{aligned}
$$

where we used the assumption of the mathematical induction, $\psi_{N-1}=\phi_{N-1}$, in the second equality. The identity $\psi_{N}=\phi_{N}$ is now equivalent to

$$
\sum_{l=1}^{N}(-1)^{l+1} \prod_{j(\neq l)}\left(w_{l}-w_{j}+a\right) \frac{\phi_{N-1}\left(w_{1}, \cdots, w_{l-1}, w_{l+1}, \cdots, w_{N}\right)}{\phi_{N}\left(w_{1}, \cdots, w_{N}\right)}=\sum_{j=1}^{N} w_{j}+\frac{N^{2} a}{2} .
$$


From the definition (4.12) of $\phi_{N}$, we get

$$
\begin{aligned}
\phi_{N-1}\left(w_{1}, \cdots, w_{l-1}, w_{l+1}, \cdots, w_{N}\right) & =\frac{w_{l}+a / 2}{\prod_{j=1}^{l-1}\left(w_{j}-w_{l}\right) \cdot \prod_{j=l+1}^{N}\left(w_{l}-w_{j}\right)} \phi_{N}\left(w_{1}, \cdots, w_{N}\right) \\
& =\frac{w_{l}+a / 2}{(-1)^{l-1} \prod_{j(\neq l)}\left(w_{l}-w_{j}\right)} \phi_{N}\left(w_{1}, \cdots, w_{N}\right) .
\end{aligned}
$$

Substituting this equation into (4.14), one sees that it is now enough to show

$$
\sum_{l=1}^{N}\left(w_{l}+a / 2\right) \prod_{j(\neq l)}\left(1+\frac{a}{w_{l}-w_{j}}\right)=\sum_{j=1}^{N} w_{j}+\frac{N^{2} a}{2} .
$$

This is proved as follows. One notices that the left hand side can be represented by a contour integral,

$$
\frac{1}{2 \pi i a} \int_{C_{R}} d z(z+a / 2) \prod_{j=1}^{N}\left(1+\frac{a}{z-w_{j}}\right),
$$

where $C_{R}$ is a contour enclosing the origin anticlockwise with radius $R$ taken to be so large that the contour surrounds all the poles $w_{j}(1 \leq j \leq N)$ in the integrand. The contour integration can be performed as follows. Expanding the product in the integrand, one finds

$$
\begin{gathered}
\frac{1}{2 \pi i a} \int_{C_{R}} d z\left[\left(z+\frac{a}{2}\right)+\sum_{l=1}^{N} \frac{a}{z-w_{l}}\left(z+\frac{a}{2}\right)+\sum_{1 \leq l<m \leq N} \frac{a^{2}}{\left(z-w_{l}\right)\left(z-w_{m}\right)}\left(z+\frac{a}{2}\right)+\cdots\right. \\
\left.+a^{N} \prod_{j=1}^{N} \frac{1}{z-w_{j}}\left(z+\frac{a}{2}\right)\right] .
\end{gathered}
$$

Further expanding the second and third terms in the integrand in $1 / z$, we see

$$
\begin{aligned}
& \frac{a}{z-w_{l}}\left(z+\frac{a}{2}\right)=a+\frac{a\left(w_{l}+a / 2\right)}{z}+O\left(\frac{1}{z^{2}}\right), \\
& \frac{a^{2}}{\left(z-w_{l}\right)\left(z-w_{m}\right)}\left(z+\frac{a}{2}\right)=\frac{a^{2}}{z}+O\left(\frac{1}{z^{2}}\right) .
\end{aligned}
$$

The higher terms in (4.18) are of order $O\left(1 / z^{2}\right)$ and thus do not contribute to the contour integral. Hence (4.17) is calculated as

$$
\begin{aligned}
& \frac{1}{2 \pi i a} \int_{C_{R}} d z(z+a / 2) \prod_{j=1}^{N}\left(1+\frac{a}{z-w_{j}}\right) \\
& =\frac{1}{2 \pi i a} \int_{C_{R}} d z\left(\sum_{l=1}^{N} \frac{a\left(w_{l}+a / 2\right)}{z}+\sum_{1 \leq l<m \leq N} \frac{a^{2}}{z}\right) \\
& =\sum_{l=1}^{N}\left(w_{l}+\frac{a}{2}\right)+\sum_{l<m} a=\sum_{j=1}^{N} w_{j}+\frac{N^{2} a}{2},
\end{aligned}
$$

thus we obtain (4.16). 
Using the identities (4.9) and (4.10) to (3.25) and (4.8) respectively, we get

$$
\begin{aligned}
& \left\langle x \mid \Psi_{z}\right\rangle=N ! C_{z} \prod_{1 \leq j<k \leq N}\left(z_{j}-z_{k}\right) e^{i \sum_{l=1}^{N} z_{l} x}, \\
& \left\langle\Psi_{z} \mid \Phi\right\rangle=i^{-N} N ! C_{z} \prod_{1 \leq j<k \leq N}\left(z_{j}^{*}-z_{k}^{*}\right) \prod_{l=1}^{N} \frac{1}{z_{l}^{*}+i / 2} .
\end{aligned}
$$

Thus using these relations and (3.27), we find that the factor $\left\langle x \mid \Psi_{z}\right\rangle\left\langle\Psi_{z} \mid \Phi\right\rangle$ in (4.7) becomes

$$
\left\langle x \mid \Psi_{z}\right\rangle\left\langle\Psi_{z} \mid \Phi\right\rangle=N ! \prod_{\alpha=1}^{M} \frac{\left(n_{\alpha} !\right)^{2}}{n_{\alpha}} \prod_{1 \leq j<k \leq N} \frac{\left|z_{j}-z_{k}\right|^{2}}{\left|z_{j}-z_{k}-i\right|^{2}} \prod_{l=1}^{N} \frac{e^{i z_{l} x}}{i z_{l}^{*}-1 / 2},
$$

Here we used the fact that $z_{j}^{*}-z_{k}^{*}$ is the complex conjugate of $z_{j}-z_{k}$ although $z_{j}^{*}$ is not that of $z_{j}$ as mentioned below Lemma 4 . The common imaginary part $c$ of $q_{\alpha(j)}$ and $q_{\alpha(k)}$ cancels out by the subtraction.

We want to rewrite this equation in terms of $q_{\alpha}$ and $n_{\alpha}$ in (3.26). For the last factor of this equation, we easily find

$$
\prod_{l=1}^{N} \frac{e^{i z_{l} x}}{i z_{l}^{*}-1 / 2}=\prod_{\alpha=1}^{M} e^{i n_{\alpha} q_{\alpha} x} \prod_{r=1}^{n_{\alpha}} \frac{1}{i q_{\alpha}+\frac{1}{2}\left(n_{\alpha}-2 r\right)} .
$$

From (3.27) and (4.21), we know the remaining factors in (4.23) is represented by $\left|\left\langle 0 \mid \Psi_{z}\right\rangle\right|^{2}$. For this quantity, the following result was obtained in Appendix B in [28],

$$
\begin{aligned}
\left|\left\langle 0 \mid \Psi_{z}\right\rangle\right|^{2} & =N ! \prod_{\alpha=1}^{M} \frac{\left(n_{\alpha} !\right)^{2}}{n_{\alpha}} \prod_{1 \leq j<k \leq N} \frac{\left|z_{j}-z_{k}\right|^{2}}{\left|z_{j}-z_{k}-i\right|^{2}} \\
& =\frac{N !}{\prod_{\alpha=1}^{M} n_{\alpha}} \prod_{1 \leq \alpha<\beta \leq M} \frac{\left|q_{\alpha}-q_{\beta}-\frac{i}{2}\left(n_{\alpha}-n_{\beta}\right)\right|^{2}}{\left|q_{\alpha}-q_{\beta}-\frac{i}{2}\left(n_{\alpha}+n_{\beta}\right)\right|^{2}} .
\end{aligned}
$$

From (4.23)-(4.25), we get

$$
\begin{aligned}
& \left\langle x \mid \Psi_{z}\right\rangle\left\langle\Psi_{z} \mid \Phi\right\rangle \\
& =N ! \prod_{\alpha<\beta}^{M} \frac{\left|q_{\alpha}-q_{\beta}-\frac{i}{2}\left(n_{\alpha}-n_{\beta}\right)\right|^{2}}{\left|q_{\alpha}-q_{\beta}-\frac{i}{2}\left(n_{\alpha}+n_{\beta}\right)\right|^{2}} \prod_{\alpha=1}^{M} \frac{e^{i n_{\alpha} q_{\alpha} x}}{n_{\alpha}} \prod_{r=1}^{n_{\alpha}} \frac{1}{i q_{\alpha}+\frac{1}{2}\left(n_{\alpha}-2 r\right)} .
\end{aligned}
$$

We can further deform (4.26) to an expression in terms of a determinant by using the Cauchy's determinant formula,

$$
\frac{\prod_{\alpha<\beta}^{M}\left(a_{\alpha}-a_{\beta}\right)\left(b_{\alpha}-b_{\beta}\right)}{\prod_{\alpha, \beta=1}^{M}\left(a_{\alpha}-b_{\beta}\right)}=(-1)^{\frac{M(M-1)}{2}} \operatorname{det}\left(\frac{1}{a_{\alpha}-b_{\beta}}\right),
$$


and a few basic properties of determinant. We find

$$
\begin{aligned}
& \left\langle x \mid \Psi_{z}\right\rangle\left\langle\Psi_{z} \mid \Phi\right\rangle \\
= & 2^{M} N ! \operatorname{det}\left(\frac{1}{n_{j}+n_{k}+2 i\left(q_{j}-q_{k}\right)}\right)_{j, k=1}^{M} \prod_{\alpha=1}^{M} e^{i n_{\alpha} q_{\alpha} x} \prod_{r=1}^{n_{\alpha}} \frac{1}{i q_{\alpha}+\frac{1}{2}\left(n_{\alpha}-2 r\right)} \\
= & 2^{M} N ! \prod_{\alpha=1}^{M}\left(\int_{0}^{\infty} d \omega_{\alpha}\right) \operatorname{det}\left(e^{-\omega_{j}\left(n_{j}+n_{k}+2 i\left(q_{j}-q_{k}\right)\right)}\right)_{j, k=1}^{M} \prod_{\alpha=1}^{M} e^{i n_{\alpha} q_{\alpha} x} \prod_{r=1}^{n_{\alpha}} \frac{1}{i q_{\alpha}+\frac{1}{2}\left(n_{\alpha}-2 r\right)} \\
= & 2^{M} N ! \prod_{\alpha=1}^{M}\left(\int_{0}^{\infty} d \omega_{\alpha}\right) \operatorname{det}\left(e^{i n_{j} q_{j} x-n_{j}\left(\omega_{j}+\omega_{k}\right)-2 i q_{j}\left(\omega_{j}-\omega_{k}\right)} \prod_{r=1}^{n_{j}} \frac{1}{i q_{j}+\frac{1}{2}\left(n_{j}-2 r\right)}\right)_{j, k=1}^{M},
\end{aligned}
$$

where in the last equality, we used a simple fact

$$
\operatorname{det}\left(a_{j}^{b_{j}+b_{k}}\right)=\operatorname{det}\left(\left(a_{j} a_{k}\right)^{b_{j}}\right) .
$$

\subsection{Fredholm determinant representation of the generating func- tion}

From (3.28), (4.7), and (4.28), we obtain an expression of $\left\langle Z^{N}(x, t)\right\rangle$ in terms of the determinant,

$$
\begin{aligned}
& \left\langle Z^{N}(x, t)\right\rangle e^{\frac{N t}{24}+\frac{N x^{2}}{2 t}} \\
= & \sum_{M=1}^{N} \frac{2^{M} N !}{M !} \prod_{\alpha=1}^{M}\left(\sum_{n_{\alpha}=1}^{\infty} \int_{\mathbb{R}-i c} \frac{d q_{\alpha}}{2 \pi} e^{-\frac{t}{2} n_{\alpha} q_{\alpha}^{2}+\frac{t}{24} n_{\alpha}^{3}} \int_{0}^{\infty} d \omega_{\alpha}\right) \delta_{\sum_{\beta=1}^{M} n_{\beta}, N} \\
\times \operatorname{det}\left(\frac{e^{i n_{j} q_{j} x+n_{j} \frac{x^{2}}{2 t}-n_{j}\left(\omega_{j}+\omega_{k}\right)-2 i q_{j}\left(\omega_{j}-\omega_{k}\right)}}{\prod_{r=1}^{n_{j}} i q_{j}+\frac{1}{2}\left(n_{j}-2 r\right)}\right)_{j, k=1}^{M} & \sum_{M=1}^{N} \frac{N !}{M !} \prod_{\alpha=1}^{M}\left(\int_{0}^{\infty} d \omega_{\alpha} \sum_{n_{\alpha}=1}^{\infty}\right) \delta_{\sum_{\beta=1}^{M} n_{\beta}, N} \times \operatorname{det}\left(\int_{\mathbb{R}-i c} \frac{d q}{\pi} \frac{e^{i n_{j} q_{j} x+n_{j} \frac{x^{2}}{2 t}-\frac{t}{2} n_{j} q^{2}+\frac{t}{24} n_{j}^{3}-n_{j}\left(\omega_{j}+\omega_{k}\right)-2 i q\left(\omega_{j}-\omega_{k}\right)}}{\prod_{r=1}^{n_{j}} i q+\frac{1}{2}\left(n_{j}-2 r\right)}\right)_{j, k=1}^{M} .
\end{aligned}
$$

The imaginary part $c$ of the contour of $q$ is discussed below (4.7) and we find it is sufficient to satisfy the condition $c>N / 2+\max _{\alpha} n_{\alpha} / 2$. Note that in (4.30), the contour satisfying

this condition passes below the poles $q=j-n_{\alpha} / 2\left(j=0,1, \cdots,\left[n_{\alpha} / 2\right]\right)$ where $[a]$ is the largest integer which is smaller than $a$ and that we can deform the contour as long as it satisfies this property. Thus in (4.30), we can relax the condition to $c>\max _{\alpha} n_{\alpha} / 2$. 
Substituting this equation into (2.5), we eventually get the Fredholm determinant representation of the generating function (2.6). Remembering (3.18), we have

$$
\begin{aligned}
& G_{\gamma_{t}}(s ; X) \\
& =1+\sum_{N=1}^{\infty} \sum_{M=1}^{N} \frac{\left(-e^{-\gamma t s}\right)^{N}}{M !} \prod_{\alpha=1}^{M}\left(\int_{0}^{\infty} d \omega_{\alpha} \sum_{n_{\alpha}=1}^{\infty}\right) \delta_{\sum_{\beta=1}^{M} n_{\beta}, N} \\
& \times \operatorname{det}\left(\int_{\mathbb{R}-i c} \frac{d q}{\pi} \frac{e^{2 i n_{j} \gamma_{t}^{2} q X+n_{j} \gamma_{t} X^{2}-\gamma_{t}^{3} n_{j} q^{2}+\frac{\gamma_{t}^{3}}{12} n_{j}^{3}-n_{j}\left(\omega_{j}+\omega_{k}\right)-2 i q\left(\omega_{j}-\omega_{k}\right)}}{\prod_{r=1}^{n_{j}}\left(i q+\frac{1}{2}\left(n_{j}-2 r\right)\right)}\right)_{j, k=1}^{M} \\
& =\sum_{M=0}^{\infty} \frac{(-1)^{M}}{M !} \prod_{\alpha=1}^{M} \int_{0}^{\infty} d \omega_{\alpha} \\
& \quad \times \operatorname{det}\left(\sum_{n=1}^{\infty}(-1)^{n-1} \int_{\mathbb{R}-i c_{n}} \frac{d q}{\pi} \frac{e^{2 i n q \gamma_{t}^{2} X+n \gamma_{t} X^{2}-\gamma_{t}^{3} n q^{2}+\frac{\gamma_{t}^{3}}{12} n^{3}-n\left(\omega_{j}+\omega_{k}\right)-2 i q\left(\omega_{j}-\omega_{k}\right)-\gamma_{t} n s}}{\prod_{r=1}^{n}\left(i q+\frac{1}{2}(n-2 r)\right)}\right)_{j, k=1}^{M},
\end{aligned}
$$

with $c_{n}>n / 2$. Shifting the variable $q$ to $q+i X / \gamma_{t}$, we get

$$
\begin{aligned}
& G_{\gamma_{t}}(s ; X) \\
& =\sum_{M=0}^{\infty} \frac{(-1)^{M}}{M !} \prod_{k=1}^{M} \int_{0}^{\infty} d \omega_{k} \\
& \quad \times \operatorname{det}\left(\sum_{n=1}^{\infty}(-1)^{n-1} \int_{\mathbb{R}-i c_{n}} \frac{d q}{\pi} \frac{e^{-n\left(\omega_{j}+\omega_{k}\right)-2 i q\left(\omega_{j}-\omega_{k}\right)-\gamma_{t}^{3} n q^{2}+\frac{\gamma_{t}^{3}}{12} n^{3}-\gamma_{t} n s}}{\prod_{r=1}^{n}\left(i q-\frac{X}{\gamma_{t}}+\frac{1}{2}(n-2 r)\right)}\right)_{j, k=1}^{M},
\end{aligned}
$$

with $c_{n}>X / \gamma_{t}+n / 2$. Applying the relation

$$
\prod_{r=1}^{n} \frac{1}{i q-X / \gamma_{t}+\frac{1}{2}(n-2 r)}=\frac{\Gamma\left(i q-\frac{X}{\gamma_{t}}-\frac{n}{2}\right)}{\Gamma\left(i q-\frac{X}{\gamma_{t}}+\frac{n}{2}\right)}
$$

to $(4.32)$, we finally obtain $(2.6)$.

\section{$5 \quad$ Another expression of the kernel}

In this section we give a derivation of the expression (2.10) in Proposition 2. We use the following two relations,

\section{Lemma 6}


(a) We set $a \in \mathbb{R}$ and $m, n \geq 0$. When $\operatorname{Im} q<-n / 2+a$, we have

$$
\frac{\Gamma\left(i q+a-\frac{n}{2}\right)}{\Gamma\left(i q+a+\frac{n}{2}\right)} e^{\frac{m^{3} n^{3}}{3}}=\int_{-\infty}^{\infty} d y \operatorname{Ai}_{\Gamma}^{\Gamma}\left(y, \frac{1}{2 m}, i q+a\right) e^{m n y},
$$

where

$$
\operatorname{Ai}_{\Gamma}^{\Gamma}(a, b, c)=\frac{1}{2 \pi} \int_{\Gamma_{i \frac{c}{b}}} d z e^{i a z+i z^{3} / 3} \frac{\Gamma(i b z+c)}{\Gamma(-i b z+c)}
$$

with $\Gamma_{p}$ defined in (2.12) below.

(b) For $u, v, x \in \mathbb{R}$ and $w \geq 0$, we have

$$
\begin{aligned}
& \frac{1}{2 \pi} \int_{-\infty}^{\infty} d p \operatorname{Ai}_{\Gamma}^{\Gamma}\left(p^{2}+v, w, i w p+u\right) e^{i p x} \\
& =\frac{1}{2^{\frac{1}{3}}} \operatorname{Ai}^{\Gamma}\left(2^{-\frac{2}{3}}(v+x), 2^{\frac{1}{3}} w, u\right) \operatorname{Ai}_{\Gamma}\left(2^{-\frac{2}{3}}(v-x), 2^{\frac{1}{3}} w, u\right),
\end{aligned}
$$

where $\operatorname{Ai}^{\Gamma}(a, b, c)$ and $\operatorname{Ai}_{\Gamma}(a, b, c)$ are defined by (2.11) and (2.12) respectively.

\section{Proof}

(a) The right hand side of (5.1) is written as

$$
\frac{1}{2 \pi} \int_{-\infty}^{\infty} d y \int_{\mathbb{R}+i m n} d z \frac{\Gamma\left(i \frac{z}{2 m}+i q+a\right)}{\Gamma\left(-i \frac{z}{2 m}+i q+a\right)} e^{i(z-i m n) y+i \frac{z^{3}}{3}} .
$$

Here we used the fact that the contour of $z$ can be deformed from $\Gamma_{2 m(i a-q)}$ to $\mathbb{R}+i m n$ since the imaginary part of the poles $2 m(i a-q+i r), r=0,1,2, \cdots$ of the integrand is larger than $m n$ when the condition $\operatorname{Im} q<a-n / 2$ is satisfied. In this equation, we change the variable $z$ on the right hand side to $y_{2}=z-i m n$ and get

$$
\begin{aligned}
& \frac{1}{2 \pi} \int_{-\infty}^{\infty} d y \int_{-\infty}^{\infty} d y_{2} \frac{\Gamma\left(i q+a-\frac{n}{2}+i \frac{y_{2}}{2 m}\right)}{\Gamma\left(i q+a+\frac{n}{2}-i \frac{y_{2}}{2 m}\right)} e^{i y y_{2}+i \frac{\left(y_{2}+i m n\right)^{3}}{3}} \\
& =\int_{-\infty}^{\infty} d y_{2} \delta\left(y_{2}\right) \frac{\Gamma\left(i q+a-\frac{n}{2}+i \frac{y_{2}}{2 m}\right)}{\Gamma\left(i q+a+\frac{n}{2}-i \frac{y_{2}}{2 m}\right)} e^{i \frac{\left(y_{2}+i m n\right)^{3}}{3}}=\frac{\Gamma\left(i q+a-\frac{n}{2}\right)}{\Gamma\left(i q+a+\frac{n}{2}\right)} e^{\frac{m^{3} n^{3}}{3}} .
\end{aligned}
$$

(b) The left hand side of (5.3) reads

$$
\frac{1}{(2 \pi)^{2}} \int_{-\infty}^{\infty} d p \int_{\Gamma_{-p+i \frac{u}{w}}} d z e^{i\left(p^{2}+v\right) z+i \frac{z^{3}}{3}+i p x} \frac{\Gamma(i w(z+p)+u)}{\Gamma(-i w(z-p)+u)}
$$

By applying the change of variables $p=\left(z_{1}-z_{2}\right) / 2^{2 / 3}$ and $z=\left(z_{1}+z_{2}\right) / 2^{2 / 3}$, we obtain the desired expression. 
Using Lemma 6, we can obtain (2.10). Applying (a) of the lemma with $a=-X / \gamma_{t}$ and $m=\gamma_{t} / 2^{2 / 3}$ to $(2.7)$, one has

$$
\begin{gathered}
K_{X}\left(\omega_{j}, \omega_{k}\right)=\sum_{n=1}^{\infty}(-1)^{n-1} \int_{\mathbb{R}-i c_{n}} \frac{d q}{\pi} \int_{-\infty}^{\infty} d y_{1} \operatorname{Ai}_{\Gamma}^{\Gamma}\left(y_{1}, \frac{1}{2^{\frac{1}{3}} \gamma_{t}}, i q-\frac{X}{\gamma_{t}}\right) e^{-2 i q\left(\omega_{j}-\omega_{k}\right)} \\
\times e^{-n\left(\omega_{j}+\omega_{k}+\gamma_{t}^{3} q^{2}+\gamma_{t} s-2^{-\frac{2}{3}} \gamma_{t} y_{1}\right)}
\end{gathered}
$$

At this point, the contour of $q$ can be replaced to $\mathbb{R}$ since since the contour of $z$ in the definition of $A_{\Gamma}^{\Gamma}(a, b, c)$ (5.2) pass below the singularity of the Gamma function. Changing the variables $q, \omega_{j}$ and $y_{1}$ to $p=2^{1 / 3} \gamma_{t} q, \xi_{j}=2 \gamma_{t}^{-1} \omega_{j}$ and $y=y_{1} / 2^{2 / 3}-p^{2} / 2^{2 / 3}-\left(\xi_{j}+\xi_{k}\right) / 2$, we see $K_{X}\left(\omega_{j}, \omega_{k}\right) d \omega_{j}=\bar{K}_{X}\left(\xi_{j}, \xi_{k}\right) d \xi_{j}$, where

$$
\begin{aligned}
\bar{K}_{X}\left(\xi_{j}, \xi_{k}\right)=\frac{2^{\frac{1}{3}}}{2 \pi} \int_{-\infty}^{\infty} d y \int_{-\infty}^{\infty} d p \mathrm{Ai}_{\Gamma}^{\Gamma}\left(p^{2}\right. & \left.+2^{\frac{2}{3}} y+\frac{\xi_{j}+\xi_{k}}{2^{\frac{1}{3}}}, \frac{1}{2^{\frac{1}{3}} \gamma_{t}}, \frac{i p-2^{\frac{1}{3}} X}{2^{\frac{1}{3}} \gamma_{t}}\right) e^{-i \frac{\left(\xi_{j}-\xi_{k}\right)}{2^{\frac{1}{3}}} p} \\
& \times \sum_{n=1}^{\infty}(-1)^{n-1} e^{-\gamma_{t} n(s-y)}
\end{aligned}
$$

Thus we can apply (2) of Lemma 6 to this equation with $v=2^{2 / 3} y+\left(\xi_{j}+\xi_{k}\right) / 2^{1 / 3}$, $w=1 /\left(2^{1 / 3} \gamma_{t}\right), u=-X / \gamma_{t}, x=\left(\xi_{j}-\xi_{k}\right) / 2^{1 / 3}$ and arrive at $(2.10)$.

\section{Multi-point distribution}

\subsection{Generating function}

We consider the $n$-point generating function defined by (2.24) and derive the result (2.25). Eq. (2.24) can be expanded in terms of the replica partition function $\left\langle Z\left(x_{l_{1}}, t\right) \cdots Z\left(x_{l_{N}}, t\right)\right\rangle$,

$$
\begin{aligned}
G_{\gamma_{t}}\left(\{s\}_{n},\{X\}_{n}\right)=1 & +\sum_{N=1}^{\infty} \frac{(-1)^{N} e^{\frac{t N}{24}}}{N !} \\
& \times \sum_{l_{1}, \cdots, l_{N}=1}^{n} e^{-\gamma_{t} \sum_{j=1}^{N}\left(s_{j}-X_{j}^{2}\right)}\left\langle Z\left(2 \gamma_{t}^{2} X_{l_{1}}, t\right) \cdots Z\left(2 \gamma_{t}^{2} X_{l_{N}}, t\right)\right\rangle .
\end{aligned}
$$

As we discussed in Sec. 3 and 4, we can express the replica partition function in terms of

the Bethe states (3.25)-(3.28) of the $\delta$-Bose gas. Specifically we can the generalize (4.7) to the case where $x^{\prime}$ s are distinct and we get

$$
\begin{aligned}
& \left\langle Z\left(x_{l_{1}}, t\right) \cdots Z\left(x_{l_{N}}, t\right)\right\rangle \\
& =\sum_{M=1}^{N} \frac{1}{M !} \prod_{\alpha=1}^{M}\left(\int_{\mathbb{R}-i c} \frac{d q_{\alpha}}{2 \pi} \sum_{n_{\alpha}=1}^{\infty}\right) \delta_{\sum_{\beta=1}^{M} n_{\beta}, N}\left\langle x_{l_{1}}, \cdots, x_{l_{N}} \mid \Psi_{z}\right\rangle\left\langle\Psi_{z} \mid \Phi\right\rangle e^{-E_{z} t} .
\end{aligned}
$$


Thus the generating function is written as

$$
G_{\gamma_{t}}\left(\{s\}_{n},\{X\}_{n}\right)=1+\sum_{N=1}^{\infty} \frac{(-1)^{N} e^{\frac{t N}{24}}}{N !} \prod_{\alpha=1}^{M}\left(\int_{\mathbb{R}-i c} \frac{d q_{\alpha}}{2 \pi} \sum_{n_{\alpha}=1}^{\infty}\right) \delta_{\sum_{\beta=1}^{M} n_{\beta}, N} A_{z} B_{z}
$$

where

$$
\begin{aligned}
& A_{z}=e^{-E_{z} t}\left\langle 0 \mid \Psi_{z}\right\rangle\left\langle\Psi_{z} \mid \Phi\right\rangle, \\
& B_{z}=\sum_{l_{1}, \cdots, l_{N}=1}^{n} e^{-\gamma_{t} \sum_{j=1}^{n}\left(s_{l_{j}}-X_{l_{j}}^{2}\right)} \frac{\left\langle 2 \gamma_{t}^{2} X_{l_{1}}, \cdots, 2 \gamma_{t}^{2} X_{l_{N}} \mid \Psi_{z}\right\rangle}{\langle 0 \mid \Psi\rangle} .
\end{aligned}
$$

Note that in two factors $A_{z}$ and $B_{z}$, only $A_{z}$ depends on the initial condition and we can readily use (3.28) and (4.28) for $A_{z}$. On the other hand, the factor $B_{z}$, which is irrelevant to specific initial conditions, has already appeared in the narrow wedge initial condition and the following approximation has been proposed in [33, 34].

For $B_{z}$, we have to estimate the equation with the following type,

$$
\sum_{l_{1}, \cdots, l_{N}=1}^{n}\left\langle x_{l_{1}}, \cdots, x_{l_{N}} \mid \Psi_{z}\right\rangle \prod_{j=1}^{N} e^{g_{l_{j}}}
$$

Here $g_{j}(j=1, \cdots, n)$ are variables which depend on $j$. Although the eigenfunction $\left\langle x_{l_{1}}, \cdots, x_{l_{N}} \mid \Psi_{z}\right\rangle$ is given in (3.25), the following compact form given in [28] is convenient for our current discussion,

$$
\left\langle y_{1}, \cdots, y_{N} \mid \Psi_{z}\right\rangle=\sum_{P \in S_{N}}^{\prime} A_{P}\left(y_{1}, \cdots, y_{N}\right) \exp \left(i \sum_{\alpha=1}^{M} q_{\alpha} \sum_{c \in \Omega_{\alpha}(P)} y_{c}-\frac{1}{4} \sum_{\alpha=1}^{M} \sum_{c, c^{\prime} \in \Omega_{\alpha}(P)}\left|y_{c}-y_{c^{\prime}}\right|\right),
$$

where $A_{P}, \Omega_{\alpha}(P)$ and the prime in the summation are defined as follows: The amplitude $A_{p}$ is represented as

$$
A_{P}\left(y_{1}, \cdots, y_{N}\right)=\operatorname{sgn} P \cdot C_{z} \prod_{\alpha=1}^{M} \prod_{k=1}^{n_{\alpha}}(k !) \prod_{1 \leq j<k \leq N}\left(z_{P(j)}-z_{P(k)}+i \operatorname{sgn}\left(y_{j}-y_{k}\right)\right) .
$$

The cluster $\Omega_{\alpha}(P)$ is defined by $\Omega_{\alpha}(P)=\{a \mid \alpha(P(a))=\beta\}$ by use of the cluster counting function $\alpha:[1, \cdots, N] \rightarrow[1, \cdots, M]$ such that $\alpha(a)=\beta$, for $\sum_{j=1}^{\beta-1} n_{j}<a \leq \sum_{j=1}^{\beta} n_{j}$. The prime means the summation over permutations which keep the order inside each cluster, i.e., the permutations satisfying the condition $j<k \rightarrow P(j)<P(k)$ for $j$ and $k$ such that $\alpha(P(j))=\alpha(P(k))(1 \leq j, k \leq N)$.

Thus (6.6) is expressed as

$$
\sum_{l_{1}, \cdots, l_{N}=1}^{n}\left\langle x_{l_{1}}, \cdots, x_{l_{N}} \mid \Psi_{z}\right\rangle \prod_{j=1}^{N} e^{g_{l_{j}}}=\sum_{l_{1}, \cdots, l_{N}=1}^{n} \sum_{P \in S_{N}}^{\prime} A_{P}\left(x_{l_{1}}, \cdots, x_{l_{N}}\right) e^{\phi\left(\{l\}_{N}, P\right)}
$$


where $\phi\left(\{l\}_{N}, P\right)$ represents the phase

$$
\phi\left(\{l\}_{N}, P\right)=i \sum_{\alpha=1}^{M} q_{\alpha} \sum_{c \in \Omega_{\alpha}(P)} y_{c}-\frac{1}{4} \sum_{\alpha=1}^{M} \sum_{c, c^{\prime} \in \Omega_{\alpha}(P)}\left|y_{c}-y_{c^{\prime}}\right|+\sum_{j=1}^{N} g_{l_{j}}
$$

The difficulty in (6.9) is that the summations over $l_{1}, \cdots, l_{N}$ and $P$ are coupled. In [33, 34], the authors proposed an approximation that the two summations factorize,

$$
\sum_{l_{1}, \cdots, l_{N}=1}^{n} \sum_{p \in S_{N}}^{\prime} A_{P}\left(y_{l_{1}}, \cdots, y_{l_{N}}\right) e^{\phi\left(\{l\}_{N}, P\right)} \sim \sum_{l_{1}, \cdots, l_{N}=1}^{n} e^{\phi\left(\{l\}_{N}\right)} \sum_{p \in S_{N}}^{\prime} A_{P}\left(y_{l_{1}}, \cdots, y_{l_{N}}\right)
$$

where $\phi\left(\{l\}_{N}\right)=\phi\left(\{l\}_{N}, P=(1,2, \cdots, N)\right)$. Under this assumption, we can perform the two summations using the relations

$$
\sum_{P \in S_{N}}^{\prime} A_{P}\left(y_{l_{1}}, \cdots, y_{l_{N}}\right)=\left\langle 0 \mid \Phi_{z}\right\rangle
$$

which comes from the identity (4.9), and

$$
\sum_{l_{1}, \cdots, l_{N}=1}^{n} e^{\phi\left(\{l\}_{N}\right)}=\prod_{\alpha=1}^{M} e^{-\frac{1}{4} \sum_{j, k=1}^{n}\left|x_{j}-x_{k}\right| \partial_{g_{j}} \partial_{g_{k}}}\left(\sum_{l=1}^{n} e^{g_{l}+i q_{\alpha} x_{l}}\right)^{n_{\alpha}},
$$

which is obtained as (3.13) in [34]. From (6.11)-(6.13) and setting $y_{j}=2 \gamma_{t}^{2} X_{j}, g_{j}=s_{j}-X_{j}^{2}$, we obtain the approximated form of $B_{z}(6.5)$,

$$
B_{z} \sim \prod_{\alpha=1}^{M} e^{-\frac{1}{2} \sum_{j, k=1}^{n}\left|X_{j}-X_{k}\right| \partial_{s_{j}} \partial_{s_{k}}}\left(\sum_{l=1}^{n} e^{-\gamma_{t} s_{l}+\gamma_{t} X_{j}^{2}+2 i \gamma_{t}^{2} q_{\alpha} X_{l}}\right)^{n_{\alpha}} .
$$

Substituting the equation above into (6.3) and following the same procedure in Sec.4.3, we have

$$
\begin{aligned}
G_{\gamma_{t}}^{\sharp}\left(\{s\}_{n},\{X\}_{n}\right)=1 & +\sum_{M=1}^{\infty} \frac{1}{M !}\left(\prod_{\alpha=1}^{M} \int_{\mathbb{R}-i c} \frac{d q_{\alpha}}{2 \pi} \sum_{n_{\alpha}=1}^{\infty}\right) \operatorname{det}\left(\frac{1}{\frac{1}{2}\left(n_{\alpha}+n_{\beta}\right)+i\left(q_{\alpha}-q_{\beta}\right)}\right) \\
& \times \prod_{\alpha=1}^{M} \prod_{r=1}^{n_{\alpha}}\left(\frac{1}{i q_{\alpha}+\frac{1}{2}\left(n_{\alpha}-2 r\right)}\right) \\
& \times \prod_{\alpha=1}^{M} e^{\gamma_{t}^{3} n_{\alpha}^{3} / 12-\frac{1}{2} \sum_{j, k=1}^{n}\left|X_{j}-X_{k}\right| \partial_{s_{j}} \partial_{s_{k}}}\left(-e^{\frac{t q_{\alpha}^{2}}{2}} \sum_{l=1}^{n} e^{-\gamma_{t} s_{l}+\gamma_{t} X_{j}^{2}+2 i \gamma_{t}^{2} q_{\alpha} X_{l}}\right)^{n_{\alpha}} .
\end{aligned}
$$

Here the index $\sharp$ indicates that we use the approximation (6.14). We find that the equation above has a similar structure to the corresponding one in the narrow wedge case in [34]. 
All the differences are just two points: The contour of $q$ includes the imaginary term $-i c$ and the factor $\prod_{\alpha=1}^{M} \prod_{r=1}^{n_{\alpha}}\left(\frac{1}{i q_{\alpha}+\frac{1}{2}\left(n_{\alpha}-2 r\right)}\right)$ is added. Thus we can just follow Sec. 4 in [34] if we replace Eqs. (4.14) and (4.19) in [34] by (1) and (2) in Lemma 6 respectively. As a result, we find the Fredholm determinant expression of $G_{\gamma_{t}}^{\sharp}\left(\{s\}_{n},\{X\}_{n}\right)$ :

$$
G_{\gamma_{t}}^{\sharp}\left(\{s\}_{n},\{X\}_{n}\right)=\operatorname{det}(1-L),
$$

where

$$
\begin{aligned}
& L\left(z, z^{\prime}\right)=\chi_{0}(z) \chi_{0}\left(z^{\prime}\right) \exp \left(-\frac{1}{2} \sum_{j, k=1}^{n}\left|X_{j}-X_{k}\right| \partial_{s_{j}} \partial_{s_{k}}-\gamma_{t} \sum_{j=1}^{n} X_{j}^{2} \partial_{s_{j}}-\left(\partial_{z}-\partial_{z^{\prime}}\right) \sum_{j=1}^{n} X_{j} \partial_{s_{j}}\right) \\
& \times \int_{-\infty}^{\infty} d u \mathrm{Ai}^{\Gamma}\left(u+z, \frac{1}{\gamma_{t}}, 0\right) \operatorname{Ai}_{\Gamma}\left(u+z^{\prime}, \frac{1}{\gamma_{t}}, 0\right) \Phi\left(\{u-s\}_{n}\right) .
\end{aligned}
$$

Here $\chi_{s}(x)$ is given below $(2.32), \operatorname{Ai}^{\Gamma}(a, b, c)$ and $\operatorname{Ai}_{\Gamma}(a, b, c)$ are defined by (2.11) and (2.12) respectively and for $\Phi\left(\{x\}_{n}\right)$, see (2.27). This expression of the kernel corresponds to Eq. (4.25) in [34] for the narrow wedge initial condition. The only difference is that the product of the ordinary Airy functions $\operatorname{Ai}(u+z) \operatorname{Ai}\left(u+z^{\prime}\right)$ is replaced by $\operatorname{Ai}^{\mathrm{T}}(u+$ $\left.z, 1 / \gamma_{t}, 0\right) \operatorname{Ai}_{\Gamma}\left(u+z^{\prime}, 1 / \gamma_{t}, 0\right)$ in (6.17).

We can further deform the expression (6.17) following the discussion in Sec. 4.2 in [34]. The equation corresponding to Eq. (4.38) in [34] becomes

$$
\begin{aligned}
L\left(z, z^{\prime}\right)=\int_{-\infty}^{\infty} & d u_{1} \cdots d u_{n}\left\langle\mathrm{Ai}_{z^{\prime}, 1 / \gamma_{t},-X_{0} / \gamma_{t}}^{\Gamma}\left|L_{0} e^{\left(X_{0}-X_{1}\right) H}\right| u_{1}\right\rangle\left\langle u_{1}\left|e^{\left(X_{1}-X_{2}\right) H}\right| u_{2}\right\rangle \times \cdots \\
& \times\left\langle u_{n-1}\left|e^{\left(X_{n-1}-X_{n}\right) H}\right| u_{n}\right\rangle\left\langle u_{n}\left|e^{\left(X_{n}-X_{0}\right) H} L_{0}\right| \mathrm{Ai}_{\Gamma, z, 1 / \gamma_{t},-X_{0} / \gamma_{t}}\right\rangle \Phi\left(\{u-s\}_{n}\right),
\end{aligned}
$$

with $X_{0}=0$. Here $H$ is the Airy Hamiltonian given below $(2.26), \Phi\left(\{x\}_{n}\right)$ and $L_{0}$ are defined by (2.27) and (2.28) respectively. The states $\left|\mathrm{Ai}_{z, b, c}^{\Gamma}\right\rangle$ and $\left|\mathrm{Ai}_{\Gamma, z, b, c}\right\rangle$ are defined by the relations $\left\langle u \mid \mathrm{Ai}_{z, b, c}^{\Gamma}\right\rangle=\mathrm{Ai}^{\Gamma}(z+u, b, c)$ and $\left\langle u \mid \mathrm{Ai}_{\Gamma, z, b, c}\right\rangle=\mathrm{Ai}_{\Gamma}(z+u, b, c)$ respectively. In this kernel, we put $L=Q_{2} Q_{1}$, where

$$
\begin{aligned}
Q_{2}\left(z^{\prime}, u_{1}\right)= & \left\langle\operatorname{Ai}_{z^{\prime}, 1 / \gamma_{t},-X_{0} / \gamma_{t}}^{\Gamma}\left|L_{0} e^{-X_{1} H}\right| u_{1}\right\rangle, \\
Q_{1}\left(u_{1}, z\right)= & \int_{-\infty}^{\infty} d u_{2} \cdots d u_{n}\left\langle u_{1}\left|e^{\left(X_{1}-X_{2}\right) H}\right| u_{2}\right\rangle \times \cdots \times\left\langle u_{n-1}\left|e^{\left(X_{n-1}-X_{n}\right) H}\right| u_{n}\right\rangle \\
& \times\left\langle u_{n}\left|e^{X_{n} H} L_{0}\right| \operatorname{Ai}_{\Gamma, z, 1 / \gamma_{t},-X_{0} / \gamma_{t}}\right\rangle \Phi\left(\{u-s\}_{n}\right) .
\end{aligned}
$$

Noticing the relation of the Fredholm determinant, $\operatorname{det}\left(1-Q_{2} Q_{1}\right)=\operatorname{det}\left(1-Q_{1} Q_{2}\right)$ and the biorthogonality (A.2) of the deformed Airy function, we get $\left.G_{\gamma_{t}}^{\sharp}\{s\}_{n},\{X\}_{n}\right)=\operatorname{det}(1-Q)$ where

$$
\begin{aligned}
& Q(x, y):=Q_{1} Q_{2}(x, y) \\
& =\int_{-\infty}^{\infty} d u_{2} \cdots d u_{n}\left\langle u_{1}\left|e^{\left(X_{1}-X_{2}\right) H}\right| u_{2}\right\rangle \cdots\left\langle u_{n}\left|e^{X_{n} H} L_{0}^{2} e^{-X_{1} H}\right| y\right\rangle \Phi\left(\{u-s\}_{n}\right) .
\end{aligned}
$$


The factor $\left\langle x\left|e^{\left(X_{j}-X_{k}\right) H}\right| y\right\rangle$ is represented in terms of the deformed Airy functions as (2.29), which will be shown in Appendix. Using (A.2) and (ii) in the Appendix, we find $L_{0}^{2}=$ $L_{0}$ and $L_{0} e^{-X_{1} H}=e^{-X_{1} H} L_{1}$, and thus we finally obtain (2.26). The second last factor $\left\langle x\left|e^{t H} L_{j}\right| y\right\rangle$ in $(2.26)$ is written as

$$
\left\langle x\left|e^{t H} L_{j}\right| y\right\rangle=\int_{0}^{\infty} d z e^{-t z} \operatorname{Ai}_{\Gamma}\left(x+z, \frac{1}{\gamma_{t}}, \frac{-X_{j}+t}{\gamma_{t}}\right) \operatorname{Ai}^{\Gamma}\left(y+z, \frac{1}{\gamma_{t}}, \frac{-X_{j}}{\gamma_{t}}\right) .
$$

This readily follows for $t<0$ from (A.4) but one has to be careful when $t>0$. The integrand is divergent as $z \rightarrow-\infty$ and hence $\left\langle x\left|e^{t H}\right| y\right\rangle$ in (2.29) is not well-defined. But in $\left\langle x\left|e^{t H} L_{j}\right| y\right\rangle, L_{j}$ projects the range of integration to the positive side only and (6.22) is still valid.

\subsection{Long-time limit}

Here we consider the long-time limit of the generating function $G_{\gamma_{t}}^{\sharp}\left(\{s\}_{n},\{X\}_{n}\right)(2.26)$. We first introduce the limiting version of $L_{j}(x, y)(2.28)$,

$$
\mathcal{L}_{j}(x, y):=\lim _{\gamma_{t} \rightarrow \infty} L_{j}(x, y)=\int_{0}^{\infty} d w \mathrm{Ai}^{-}\left(x+w, X_{j}\right) \mathrm{Ai}^{+}\left(y+w, X_{j}\right),
$$

where $\mathrm{Ai}^{\mp}\left(x+w, X_{j}\right)$ is the limit of the Gamma-deformed Airy functions (2.11) and (2.12),

$$
\begin{aligned}
& \operatorname{Ai}^{-}(x, y):=\lim _{\gamma_{t} \rightarrow \infty} \frac{1}{\gamma_{t}} \operatorname{Ai}^{\Gamma}\left(x, \frac{1}{\gamma_{t}}, \frac{-y}{\gamma_{t}}\right)=\frac{1}{2 \pi} \int_{\Gamma_{-i y}} d z \frac{e^{i z x+\frac{i z^{3}}{3}}}{i z-y} \\
& \operatorname{Ai}^{+}(x, y):=\lim _{\gamma_{t} \rightarrow \infty} \gamma_{t} \operatorname{Ai}_{\Gamma}\left(x, \frac{1}{\gamma_{t}}, \frac{-y}{\gamma_{t}}\right)=\frac{1}{2 \pi} \int_{-\infty}^{\infty} d z e^{i z x+\frac{i z^{3}}{3}}(-i z-y) .
\end{aligned}
$$

Here the contour $\Gamma_{-i y}$ in (6.24) is given below (2.12). Noticing

$$
\lim _{t \rightarrow \infty} \Phi\left(\{u-s\}_{n}\right)=1-\prod_{j=1}^{n}\left(1-\Theta\left(s_{j}-u_{j}\right)\right)
$$

where $\Theta(x)=1(x \geq 0), 0(x<0)$, we find the long-time limit of $(2.26)$ becomes

$$
\lim _{t \rightarrow \infty} G_{\gamma_{t}}^{\sharp}\left(\{s\}_{n},\{X\}_{n}\right)=\operatorname{det}\left(1-\mathcal{L}_{1}+\bar{P}_{s_{1}} e^{\left(X_{1}-X_{2}\right) H} \cdots \bar{P}_{s_{n-1}} e^{\left(X_{n-1}-X_{n}\right) H} \bar{P}_{s_{n}} e^{\left(X_{n}-X_{1}\right) H} \mathcal{L}_{1}\right) .
$$

Here $\bar{P}_{s}$ represents projection operator onto $(-\infty, s)$. To this equation, we apply the result of Appendix in [34] with $K_{i j}^{0}=\delta_{i j} K$ replaced by $K_{i j}^{0}=\delta_{i j} \mathcal{L}_{j}$. We eventually obtain the Fredholm determinant with matrix kernel $M$.

$$
\lim _{t \rightarrow \infty} G_{\gamma_{t}}^{\sharp}\left(\{s\}_{n},\{X\}_{n}\right)=\operatorname{det}(1-M),
$$


where

$$
M_{j k}\left(\xi_{1}, \xi_{2}\right)= \begin{cases}\chi_{s_{j}}\left(\xi_{1}\right)\left\langle\xi_{1}\left|e^{\left(X_{j}-X_{k}\right) H} \mathcal{L}_{k}\right| \xi_{k}\right\rangle \chi_{s_{k}}\left(\xi_{2}\right), & \text { for } j \geq k \\ -\chi_{s_{j}}\left(\xi_{1}\right)\left\langle\xi_{1}\left|e^{\left(X_{j}-X_{k}\right) H}\left(1-\mathcal{L}_{k}\right)\right| \xi_{2}\right\rangle \chi_{s_{k}}\left(\xi_{2}\right), & \text { for } j<k\end{cases}
$$

Here $\chi_{s}(x)$ is given in $(2.34)$ below.

We find that this matrix kernel is equivalent to $\mathcal{K}_{12}\left(X_{j}, \xi_{1} ; X_{k}, \xi_{2}\right)$. By taking the limit $\gamma_{t} \rightarrow \infty$ in (6.22), we easily find the factor $\left\langle\xi_{1}\left|e^{\left(X_{j}-X_{k}\right) H} \mathcal{L}_{k}\right| \xi_{k}\right\rangle$ in the case $j \geq k$ of $(6.29)$ is expressed as

$$
\begin{aligned}
& \left\langle\xi_{1}\left|e^{\left(X_{j}-X_{k}\right) H} \mathcal{L}_{k}\right| \xi_{2}\right\rangle \\
& =\int_{0}^{\infty} d z e^{-\left(X_{j}-X_{k}\right) z} \mathrm{Ai}^{-}\left(\xi_{1}+z, X_{j}\right) \mathrm{Ai}^{+}\left(\xi_{2}+z, X_{k}\right) \\
& =\int_{-\infty}^{\infty} d w_{1} \int_{-\infty}^{\infty} d w_{2} e^{i w_{1} \xi_{1}+i w_{2} \xi_{2}+\frac{i}{3}\left(w_{1}^{3}+w_{2}^{3}\right)} \frac{i w_{2}+X_{k}}{\left(i w_{1}-X_{j}\right)\left(i w_{1}+i w_{2}-X_{j}+X_{k}\right)}
\end{aligned}
$$

Here we used (6.24) and (6.25) in the second equality. Noticing

$$
\frac{i w_{2}+X_{k}}{\left(i w_{1}-X_{j}\right)\left(i w_{1}+i w_{2}-X_{j}+X_{k}\right)}=\frac{1}{i w_{1}-X_{j}}-\frac{1}{i w_{1}+i w_{2}-X_{j}+X_{k}},
$$

we finally find the factor can be represented as the Airy function. For $j \geq k$, we obtain

$$
\begin{aligned}
\left\langle\xi_{1}\left|e^{\left(X_{j}-X_{k}\right) H} \mathcal{L}_{k}\right| \xi_{2}\right\rangle & =\int_{0}^{\infty} d w e^{-w\left(X_{j}-X_{k}\right)} \operatorname{Ai}\left(\xi_{1}+w\right) \operatorname{Ai}\left(\xi_{2}+w\right) \\
& +\operatorname{Ai}\left(\xi_{2}\right)\left(e^{-X_{j}^{3}+X_{j} \xi_{1}}-\int_{0}^{\infty} d w e^{-X_{j} w} \operatorname{Ai}\left(\xi_{1}+w\right)\right) .
\end{aligned}
$$

Similarly for the case $j<k$ in (6.29), we get

$$
\begin{aligned}
\left\langle\xi_{1}\left|e^{\left(X_{j}-X_{k}\right) H} \mathcal{L}_{k}\right| \xi_{2}\right\rangle & =-\int_{-\infty}^{0} d w e^{-w\left(X_{j}-X_{k}\right)} \operatorname{Ai}\left(\xi_{1}+w\right) \operatorname{Ai}\left(\xi_{2}+w\right) \\
& +\operatorname{Ai}\left(\xi_{2}\right)\left(e^{-X_{j}^{3}+X_{j} \xi_{1}}-\int_{0}^{\infty} d w e^{-X_{j} w} \operatorname{Ai}\left(\xi_{1}+w\right)\right) .
\end{aligned}
$$

Hence we finally find $M_{j k}\left(\xi_{1}, \xi_{2}\right)=\mathcal{K}_{12}\left(X_{j}, \xi_{1} ; X_{k}, \xi_{2}\right)$.

\section{Directed polymer interpretation}

Our result can also be rephrased for the free energy distribution of the $1+1$ dimensional

directed polymer in random media. First let us rewrite the path integral expression of $Z_{\nu, \lambda, D}(x, t)(3.8)$ as

$$
Z_{\beta, \gamma, D}(x, t)=\int_{0}^{\infty} d y \int_{x(0)=y}^{x(t)=x} D[x(\tau)] \exp [-\beta(H[x]+\mu B(y))],
$$


where

$$
H[x]=\int_{0}^{t} d \tau\left(\frac{\gamma}{2}\left(\frac{d x}{d \tau}\right)^{2}-\sqrt{D} \eta(x(\tau), \tau)\right) .
$$

The parameters $\beta, \gamma$ and $\mu$ are defined as

$$
\beta=\frac{\lambda}{2 \nu}, \quad \gamma=\frac{1}{\lambda}, \quad \mu=\frac{\alpha}{\beta}=\sqrt{\frac{D}{2 \nu}}
$$

in terms of those of the KPZ equation (1.1).

This expression (7.1) can be interpreted as the partition function of a directed polymer in random media with inverse temperature $\beta$. On the two dimensional $(x, \tau) \quad(x \in \mathbb{R}$ and $\tau \geq 0)$ plane the configuration of a polymer is represented as a function $x(\tau) \quad(0 \leq \tau \leq t)$. Here it is assumed that $x(\tau)$ is a single-valued function, which means that the polymer is "directed" for $\tau$ coordinate. For a configuration $x(\tau) \quad(0 \leq \tau \leq t)$ the bulk energy $H[x](7.2)$ is assigned. The first term of $H[x]$ represents the elastic energy of the polymer whose strength is determined by $\gamma$, and the second term represents a random potential energy with $D$ adjusting its strength.

The half Brownian motion initial condition (1.4) in the KPZ equation corresponds to the following boundary condition in the language of the random directed polymer. At the boundary $\tau=0$, the position of the polymer $x(0)$ can take any positive value $y \geq 0$ and, depending on the position $y$, the random boundary energy $\mu B(y)$ is assigned to the polymer. Note that the other edge $x(t)$ is pinned at $x$. As a statistical mechanical model, $\mu$ in (7.1) is an independent parameter, but when translated from the KPZ equation one has to keep in mind that it is written as $\mu=\sqrt{\beta \gamma D}$.

We want to discuss the low temperature behavior of the directed polymer for a fixed $t$. When the temperature is sufficiently low, the configuration which gives the minimum energy is dominant for the free energy. When the position $x$ of the edge $x(t)$ of the polymer is positive, the other position of the edge $y$ tends to be close to $x$ because otherwise the polymer obtains excess bulk elastic energy. In this case, it is dominated by the boundary energy $B(y)$. Thus in the positive region $x>0$, the free energy fluctuation is described by the Gaussian. On the other hand, when $x<0$, the edge $x(0)$ tends to be pinned at the origin. In this case, the free energy fluctuation is determined only by the bulk energy (note that $B(y=0)=0)$. In between around $x=0$ one observes the crossover distribution, which is exactly what have been computed in this paper.

Concretely, from $(3.3),-h_{\nu, \lambda, D}$ corresponds to the free energy $f_{\beta, \gamma, D}(x, t)$ of the random directed polymer and hence (2.13) is translated as

$$
\operatorname{Prob}\left(\beta f_{\beta, \gamma, D}(x, t)-\frac{\gamma_{t}^{3}}{12}+\gamma_{t} X^{2} \geq \gamma_{t} s\right)=F_{\gamma_{t}}(s ; X)
$$

where $F_{\gamma_{t}}(x ; X)$ is given in (2.17) and $\alpha$ and $\gamma_{t}$ are written in terms of $\gamma$ and $\beta$ as $\alpha=$ $\left(\beta^{3} \gamma D\right)^{1 / 2}$ and $\gamma_{t}=\left(\beta^{5} \gamma D^{2} t / 2\right)^{1 / 3}$ respectively. Based on (7.4), one can obtain various 
information about the the free energy of the directed polymer. For instance one sees that as $\beta \rightarrow \infty$ the macroscopic free energy scales as $O\left(\beta^{4}\right)$, the fluctuations of the bulk free energy is $O\left(\beta^{2 / 3}\right)$. To get a nontrivial distribution in the low temperature limit $\beta \rightarrow \infty$, one has to scale the space direction as $X=O\left(\beta^{1 / 3}\right)$. The free energy distribution in this limit is given by (2.22) since $\beta \rightarrow \infty$ implies $\gamma_{t} \rightarrow \infty$. This describes the crossover between boundary and bulk free energy fluctuation, i.e., between Gaussian and the GUE Tracy-Widom distribution.

\section{Conclusion}

In this paper we have considered the KPZ equation (1.1) with the half Brownian motion initial condition (1.4). Using the Bethe ansatz results of the one-dimensional attractive $\delta$ Bose gas, we have obtained a Fredholm determinant expression for the generating function of exponential moments of the height (Theorem 1). Thanks to this result and Proposition 2 , the compact representation of the probability distribution of the height was obtained (Theorem 3). We have also found an expression for the multi-point generating function employing an approximation proposed in [33, 34].

These results are expressed in terms of the deformed Airy functions (2.11) and (2.12). If we change these functions to the ordinary Airy function, we recover the results for the narrow wedge initial condition obtained in [14, 15, 16, 17]. The deformed Airy functions have some nice properties, which are discussed in the Appendix. They satisfy the biorthogonality relation. Their time evolution by the Airy Hamiltonian are again given by the same functions with a parameter modified. We remark that similar relations of the multiple Hermite polynomials played an important role in the study of the PNG model with external source $[42,43]$. In the long time limit, the kernel of the Fredholm determinant becomes the rank one perturbation of the Airy kernel. It is not clear whether the higher rank perturbations studied in [47] have corresponding finite time generalizations.

\section{A Properties of the deformed Airy functions}

In this appendix, we pick up a few properties of the gamma-deformed Airy functions (2.11) and (2.12), which are necessary for the discussions in Sec. 6. Let $t \leq 0$ and $H$ be the Airy Hamiltonian $H=-\partial^{2} / \partial x^{2}+x$. We have the following relations.

(i) The deformed Airy function representation of the propagator

$$
\left\langle x\left|e^{t H}\right| y\right\rangle=\int_{-\infty}^{\infty} d z e^{-t z} \mathrm{Ai}_{\Gamma}(x+z, b, c-b t) \mathrm{Ai}^{\Gamma}(y+z, b, c) .
$$

Note that when we set $t=0$ in (A.1), we get the biorthogonality relation of the deformed Airy function,

$$
\int_{-\infty}^{\infty} d w \operatorname{Ai}_{\Gamma}(x+w, b, c) \operatorname{Ai}^{\Gamma}(y+w, b, c)=\delta(x-y) .
$$


Using (A.1), we easily get the following relations.

(ii) "Time evolution" by the Airy Hamiltonian

$$
\begin{aligned}
& e^{t H} \operatorname{Ai}^{\Gamma}(x+w, b, c)=e^{-t w} \operatorname{Ai}^{\Gamma}(x+w, b, c-b t), \\
& e^{t H} \operatorname{Ai}_{\Gamma}(x+w, b, c)=e^{-t w} \operatorname{Ai}_{\Gamma}(x+w, b, c+b t) .
\end{aligned}
$$

When we set $b=1 / \gamma_{t}$ and $c=-X / \gamma_{t}$ and take the limit $\gamma_{t} \rightarrow \infty$, these relations (A.1), (A.3) and (A.4) become those for $\mathrm{Ai}^{-}(x, y)(6.24)$ and $\mathrm{Ai}^{+}(x, y)$ (6.25),

$$
\begin{aligned}
& \left\langle x\left|e^{t H}\right| y\right\rangle=\int_{-\infty}^{\infty} d z e^{-t z} \mathrm{Ai}^{+}(x+z, X) \mathrm{Ai}^{-}(y+z, X), \\
& e^{t H} \mathrm{Ai}^{-}(x+w, X)=e^{-t w} \mathrm{Ai}^{-}(x+w, X-t), \\
& e^{t H} \mathrm{Ai}^{+}(x+w, X)=e^{-t w} \mathrm{Ai}^{+}(x+w, X+t) .
\end{aligned}
$$

\section{Proof}

(i) The factor $\left\langle x\left|e^{t H}\right| y\right\rangle$ can be represented in terms of the Airy function,

$$
\left\langle x\left|e^{t H}\right| y\right\rangle=\int_{-\infty}^{\infty} d w e^{-t w} \operatorname{Ai}(w+x) \operatorname{Ai}(w+y) .
$$

Thus it is enough to show that

$$
\int_{-\infty}^{\infty} d w e^{-t w} \operatorname{Ai}_{\Gamma}(x+w, b, c-b t) \operatorname{Ai}^{\Gamma}(y+w, b, c)=\int_{-\infty}^{\infty} d w e^{-t w} \operatorname{Ai}(w+x) \operatorname{Ai}(w+y) .
$$

In the definition (2.11) of $\operatorname{Ai}^{\Gamma}(a, b, c)$, we find that in the case $c>0$, the contour integral $\Gamma_{i c / b}$ can be replaced by $\mathbb{R}$ while in the case $c \leq 0$, we can divide the contour integral into the integral on $\mathbb{R}$ and the contributions from the poles at $(i c+m) / b, m=0,1,2 \cdots$ of $\Gamma(i b z+c)$. Thus $(2.11)$ can be represented as

$$
\operatorname{Ai}^{\Gamma}(a, b, c)= \begin{cases}\frac{1}{2 \pi} \int_{-\infty}^{\infty} d z e^{i z a+i \frac{z^{3}}{3}} \Gamma(i b z+c), & c>0, \\ \frac{1}{2 \pi} \int_{-\infty}^{\infty} d z e^{i z a+i \frac{z^{3}}{3}} \Gamma(i b z+c)+\sum_{N=0}^{n} \frac{(-1)^{N}}{N ! b} e^{-\frac{(c+N) a}{b}+\frac{(c+N)^{3}}{3 b^{3}}}, & c \leq 0 .\end{cases}
$$

Here $n$ is the maximum integer satisfying $c+n<0$.

Thus for the case $c>0$, using (2.12) and the first relation in (A.10), we find the left hand side of (A.9) is represented as

$$
\begin{aligned}
& \frac{1}{2 \pi} \int_{-\infty}^{\infty} d w \int_{-\infty}^{\infty} d z_{1} \int_{-\infty}^{\infty} d z_{2} e^{i w\left(z_{1}+z_{2}+i t\right)+i\left(z_{1} x+z_{2} y\right)+\frac{i}{3}\left(z_{1}^{3}+z_{2}^{3}\right)} \frac{\Gamma\left(i b z_{2}+c\right)}{\Gamma\left(-i b z_{1}+c-b t\right)} \\
& =\frac{1}{2 \pi} \int_{-\infty}^{\infty} d w \int_{\mathbb{R}-i t} d z_{1} \int_{-\infty}^{\infty} d z_{2} e^{i w\left(z_{1}+z_{2}+i t\right)+i\left(z_{1} x+z_{2} y\right)+\frac{i}{3}\left(z_{1}^{3}+z_{2}^{3}\right)} \frac{\Gamma\left(i b z_{2}+c\right)}{\Gamma\left(-i b z_{1}+c-b t\right)}
\end{aligned}
$$


Here we deformed the contour of $z_{1}$ to $\mathbb{R}-i t$. This deformation is valid when $t \leq 0$. Note that in this equation above, the part $z_{1}+z_{2}+i t$ becomes real. Thus one has

$$
\begin{aligned}
& \int_{\mathbb{R}-i t} d z_{1} \int_{-\infty}^{\infty} d z_{2} \delta\left(z_{1}+z_{2}+i t\right) e^{i\left(z_{1} x+z_{2} y\right)+\frac{i}{3}\left(z_{1}^{3}+z_{2}^{3}\right)} \frac{\Gamma\left(i b z_{2}+c\right)}{\Gamma\left(-i b z_{1}+c-b t\right)} \\
& =\int_{\mathbb{R}-i t} d z_{1} \int_{-\infty}^{\infty} d z_{2} \delta\left(z_{1}+z_{2}+i t\right) e^{i\left(z_{1} x+z_{2} y\right)+\frac{i}{3}\left(z_{1}^{3}+z_{2}^{3}\right)} \\
& =\frac{1}{2 \pi} \int_{-\infty}^{\infty} d w \int_{-\infty}^{\infty} d z_{1} \int_{-\infty}^{\infty} d z_{2} e^{i w\left(z_{1}+z_{2}+i t\right)+i\left(z_{1} x+z_{2} y\right)+\frac{i}{3}\left(z_{1}^{3}+z_{2}^{3}\right)} .
\end{aligned}
$$

In the second equality, the factor of the Gamma functions is eliminated because of the delta function. We easily find that the last expression of this equation is equal to the right hand side of (A.9) from the integral representation of the Airy function,

$$
\operatorname{Ai}(x)=\int_{-\infty}^{\infty} d z e^{i z x+i \frac{z^{3}}{3}}
$$

For $c<0$, on the other hand, the terms coming from the pole contributions in (A.10) are added. However we find that these terms do not affect the result since

$$
\begin{aligned}
& \int_{-\infty}^{\infty} d w e^{-t w} \operatorname{Ai}_{\Gamma}(x+w, b, c) e^{-\frac{(c+N)}{b}(y+w)} \\
= & \frac{1}{2 \pi} \int_{-\infty}^{\infty} d w \int_{-\infty}^{\infty} d z_{1} \frac{e^{i\left(z_{1}+i \frac{c+N}{b}+i t\right) w-\frac{c+N}{b} y+i x z_{1}+i \frac{z_{1}^{3}}{3}}}{\Gamma\left(-i b z_{1}+c-b t\right)} \\
= & \int_{\mathbb{R}-i\left(\frac{c+N}{b}+t\right)} d z_{1} \delta\left(z_{1}+i \frac{c+N}{b}+i t\right) \frac{e^{-\frac{c+N}{b} y+i z_{1} x}}{\Gamma\left(-i b z_{1}+c-b t\right)}=\frac{e^{\frac{c+N}{b}(x-y)+t x}}{\Gamma(-N)}=0 .
\end{aligned}
$$

(ii) Here we only consider (A.3) and omit the proof of (A.4) since it can be shown by using the same strategy as (A.3). The left hand side is given by

$$
\left\langle x\left|e^{t H}\right| \operatorname{Ai}_{w, b, c}^{\Gamma}\right\rangle=\int_{-\infty}^{\infty} d y\left\langle x\left|e^{t H}\right| y\right\rangle \operatorname{Ai}^{\Gamma}(y+w, b, c) .
$$

Substituting (A.1) into this equation, we readily obtain the right hand side of (A.3),

$$
\begin{aligned}
\left\langle x\left|e^{t H}\right| \mathrm{Ai}_{w, b, c}^{\Gamma}\right\rangle & =\int_{-\infty}^{\infty} d z \int_{-\infty}^{\infty} d y e^{-t z} \mathrm{Ai}^{\Gamma}(x+z, b, c-b t) \operatorname{Ai}_{\Gamma}(y+z, b, c) \operatorname{Ai}^{\Gamma}(y+w, b, c) \\
& =e^{-t w} \operatorname{Ai}^{\Gamma}(x+w, b, c-b t) .
\end{aligned}
$$

Here in the second equality, we used the biorthogonality relation (A.2). 


\section{Acknowledgments}

The work of T.I. and T.S. is supported by KAKENHI (22740251) and KAKENHI (22740054) respectively.

\section{References}

[1] M. Kardar, G. Parisi and Y. C. Zhang, Dynamic scaling of growing interfaces, Phys. Rev. Lett., 56: 889-892, 1986.

[2] K. Johansson, Shape fluctuations and random matrices, Commun. Math. Phys., 209: 437-476, 2000.

[3] M. Prähofer and H. Spohn, Statistical self-similarity of one-dimensional growth processes, Physica A, 279: 342-352, 2000.

[4] M. Prähofer and H. Spohn, Universal distributions for growth processes in $1+1$ dimensions and random matrices, Phys. Rev. Lett., 84: 4882-4885, 2000.

[5] C. A. Tracy and H. Widom, Level-spacing distributions and the Airy kernel, Commun. Math. Phys., 159: 151-174, 1994.

[6] M. Prähofer and H. Spohn, Scale invariance of the PNG droplet and the Airy process, J. Stat. Phys., 108: 1071-1106, 2002.

[7] T. Sasamoto, Spatial correlations of the 1D KPZ surface on a flat substrate, J. Phys. A, 38: L549-L556, 2005.

[8] A. Borodin, P. L. Ferrari, M. Prähofer and T. Sasamoto, Fluctuation properties of the TASEP with periodic initial configuration, J. Stat. Phys., 129: 1055-1080, 2007.

[9] T. Sasamoto, Fluctuations of the one-dimensional asymmetric exclusion process using random matrix techniques, J. Stat. Mech., P07007, 2007.

[10] T. Kriecherbauer, J. Krug, A pedestrian's view on interacting particle systems, KPZ universality, and random matrices, J. Phys. A, 43: 403001, 2010.

[11] P. Ferrari, From interacting particle systems to random matrices, J. Stat. Mech., P10016, 2010.

[12] T. Sasamoto and H. Spohn, The 1+1-dimensional Kardar-Parisi-Zhang equation and its universality class, J. Stat. Mech., P11013, 2010.

[13] K. A. Takeuchi and M. Sano, Universal Fluctuations of Growing Interfaces: Evidence in Turbulent Liquid Crystals, Phys. Rev. Lett., 104: 230601, 2010. 
[14] T. Sasamoto and H. Spohn, One-Dimensional Kardar-Parisi-Zhang Equation: An Exact Solution and its Universality, Phys. Rev. Lett., 104: 230602, 2010.

[15] T. Sasamoto and H. Spohn, Exact height distributions for the KPZ equation with narrow wedge initial condition, Nuc. Phys. B, 834: 523-542, 2010.

[16] T. Sasamoto and H. Spohn, The Crossover Regime for the Weakly Asymmetric Simple Exclusion Process, J. Stat. Phys., 140: 209-231, 2010.

[17] G. Amir, I. Corwin and J. Quastel, Probability distribution of the free energy of the continuum directed random polymer in $1+1$ dimensions, Com. Pure. Appl. Math., 64: 466-537, 2011.

[18] C. A. Tracy and H. Widom, Integral formulas for the asymmetric simple exclusion process, Comnum. Math. Phys., 279: 815-844, 2008.

[19] C. A. Tracy and H. Widom, Asymptotics in ASEP with step initial condition, Comnum. Math. Phys., 290: 129-154, 2009.

[20] L. Bertini and G. Giacomin, Stochastic Burgers and KPZ equations from particle systems, Commun. Math. Phys., 183: 571-607, 1997.

[21] P. Ferrari and R. Frings, Finite time correlations in KPZ growth models, J. Stat. Phys., 144: 1123-1150, 2011.

[22] J. Quastel and D. Remenik, Local Brownian property of the narrow wedge solution of the KPZ equation, Elect. Comm. in Probab., 16: 712-719, 2011.

[23] N. O'Connell, Directed polymers and the quantum Toda lattice, Ann. Prob., 40: 437-458, 2012.

[24] N. O'Connell and J. Warren, A multi-layer extension of the stochastic heat equation, arXiv: 1104.3509 .

[25] P. Goncalves and M. Jara, Universality of KPZ equation, arXiv: 1003.4478.

[26] P. Goncalves and M. Jara, Crossover to the KPZ equation, Ann. Henri Poincaré, 13: 813-826, 2012.

[27] V. Dotsenko, Bethe ansatz derivation of the Tracy-Widom distribution for onedimensional directed polymers, EPL, 90: 20003, 2010.

[28] V. Dotsenko, Replica Bethe ansatz derivation of the Tracy-Widom distribution of the free energy fluctuations in one-dimensional directed polymers, J. Stat. Mech., P07010, 2010 .

[29] P. Calabrese, P. Le Doussal and A. Rosso, Free-energy distribution of the directed polymer at high temperature, EPL, 90: 20002, 2010. 
[30] M. Kardar, Replica Bethe ansatz studies of two-dimensional interfaces with quenched random impurities, Nuc. Phys. B, 290: 582-602, 1987.

[31] E. H. Lieb, and W. Liniger, Exact analysis of an interacting Bose gas. I. the general solution and the ground state, Phys. Rev., 130: 1605-1616, 1963.

[32] J. B. McGuire, Study of exactly soluble onedimensional Nbody problems J. Math. Phys., 5: 622-636, 1964.

[33] S. Prolhac and H. Spohn, Two-point generating function of the free energy for a directed polymer in a random medium, J. Stat. Mech., P01031, 2011.

[34] S. Prolhac and H. Spohn, The one-dimensional KPZ equation and the Airy process, J. Stat. Mech., P03020, 2011.

[35] I. Corwin and J. Quastel, Renormalization fixed point of the KPZ universality class, arXiv:1103.3422.

[36] P. Calabrese, P. Le Doussal, An exact solution for the KPZ equation with flat initial conditions, Phys. Rev. Lett. 106, 250603, 2011.

[37] C. A. Tracy and H. Widom, On ASEP with step Bernoulli initial condition, J. Stat. Phys., 137, 825-838, 2009.

[38] I. Corwin and J. Quastel, Crossover distributions at the edge of the rarefaction fan, arXiv:1006.1338.

[39] F. Bornemann, On the numerical evaluation of Fredholm determinants, Math. Comp., 79: 871-915, 2010.

[40] F. Bornemann, On the numerical evaluation of distributions in random matrix theory: A review, Markov Processes Relat. Fields, 16:803-866, 2010.

[41] S. Prolhac and H. Spohn, The height distribution of the KPZ equation with sharp wedge initial condition: numerical evaluations, arXiv:1105.0383.

[42] T. Imamura and T. Sasamoto, Fluctuations of the one-dimensional polynuclear growth model with external sources, Nucl. Phys. B, 699, 503-544, 2004.

[43] T. Imamura and T. Sasamoto, Polynuclear growth model with external source and random matrix model with deterministic source, Phys. Rev. E, 71, 041606, 2005.

[44] I. Corwin, P. L. Ferrari and S. Péché, Limit processes for TASEP with shocks and rarefaction fans, J. Stat. Phys., 140, 232-267, 2010.

[45] J. Baik and E. M. Rains, Limiting distributions for a polynuclear growth model with external sources, J. Stat. Phys., 100, 523-541, 2000. 
[46] L. Bertini and N. Cancrini, The stochastic heat equation: Feynman-Kac formula and intermittence, J. Stat. Phys., 78, 1377-1401, 1995.

[47] J. Baik and G. Ben Arous and S. Péché, Phase transition of the largest eigenvalue for non-null complex sample covariance matrices, Ann. Prob., 33, 1643-1697, 2006. 